J. Noncommut. Geom. 9 (2015), 1295-1342

DOI $10.4171 / \mathrm{JNCG} / 224$
Journal of Noncommutative Geometry

(C) European Mathematical Society

\title{
Jacobi and Poisson algebras
}

\author{
Ana L. Agore*,** and Gigel Militaru**
}

\begin{abstract}
Jacobi/Poisson algebras are algebraic counterparts of Jacobi/Poisson manifolds. We introduce representations of a Jacobi algebra $A$ and Frobenius Jacobi algebras as symmetric objects in the category. A characterization theorem for Frobenius Jacobi algebras is given in terms of integrals on Jacobi algebras. For a vector space $V$ a non-abelian cohomological type object $\mathcal{J H}^{2}(V, A)$ is constructed: it classifies all Jacobi algebras containing $A$ as a subalgebra of codimension equal to $\operatorname{dim}(V)$. Representations of $A$ are used in order to give the decomposition of $\mathcal{J H}^{2}(V, A)$ as a coproduct over all Jacobi $A$-module structures on $V$. The bicrossed product $P \bowtie Q$ of two Poisson algebras recently introduced by Ni and Bai appears as a special case of our construction. A new type of deformations of a given Poisson algebra $Q$ is introduced and a cohomological type object $\mathcal{H} \mathcal{A}^{2}(P, Q \mid(\triangleleft, \triangleright,\llcorner, \rightarrow))$ is explicitly constructed as a classifying set for the bicrossed descent problem for extensions of Poisson algebras. Several examples and applications are provided.
\end{abstract}

Mathematics Subject Classification (2010). 17B63, 17B60, 17C10.

Keywords. Jacobi algebra, representations and Frobenius objects, unified products.

\section{Introduction}

Noncommutative geometry as well as quantum group theory is based on the same idea: instead of working with points on a given space $M$ which can be a compact topological group, a Lie group, an algebraic group, a manifold, etc. we can work equivalently with the algebra Fun $(M)$ of functions on $M$. The definition of the algebra of functions depends on the category of spaces that we are dealing with: for instance, if $M$ is a compact topological group then Fun $(M):=\mathcal{R}(M)$, the algebra of real-valued continuous representative functions on $M$, while if $M$ is a manifold then Fun $(M):=C^{\infty}(M)$, the algebra of all real smooth functions on $M$. Thus there exists a contravariant functor Fun $(-)$, which very often is in fact a duality of categories, between a certain category of spaces and a certain category of algebras

${ }^{*}$ A. L. Agore is Postoctoral Fellow of the Fund for Scientific Research-Flanders (Belgium) (F.W.O. Vlaanderen).

${ }^{* *}$ This work was supported by a grant of the Romanian National Authority for Scientific Research, CNCS-UEFISCDI, grant no. 88/05.10.2011. 
endowed with additional structures compatible with the algebra structure such as coalgebras (i.e. Hopf algebras), Lie brackets (i.e. Poisson algebras) etc. Adopting this categorical viewpoint, purely geometric concepts sat at the foundation of a research program started at the end of the 80's which deals with their algebraic counterparts: the functor Fun (-) being the tool through which geometrical problems can be restated and approached in algebraic language by way of a certain category of algebras. The present paper fits within this context: we deal with Jacobi algebras which are abstract algebraic counterparts of Jacobi manifolds. Jacobi manifolds, as generalizations of symplectic or more generally Poisson manifolds, were introduced independently by Kirillov [30] and Lichnerowicz [33]. A Jacobi manifold is a smooth manifold endowed with a bivector field $\Lambda$ and a vector field $E$ satisfying some compatibility conditions. When the vector field $E$ identically vanishes, the Jacobi manifold is just a Poisson manifold. Equivalently, a Jacobi manifold is a smooth manifold $M$ such that the commutative algebra $A:=C^{\infty}(M)$ of real smooth functions on $M$ is endowed with a Lie bracket $[-,-]$ such that $[a b, c]=a[b, c]+[a, c] b-a b\left[1_{A}, c\right]$, for all $a, b, c \in A$. Such an algebraic object $A$ is called a Jacobi algebra while a Poisson algebra is just a Jacobi algebra such that $\left[1_{A}, a\right]=0$, for all $a \in A$. Poisson algebras appear in several areas of research situated at the border between mathematics and physics such as: Hamiltonian mechanics, differential geometry, Lie groups and representation theory, noncommutative algebraic/diferential geometry, (super)integrable systems, quantum field theory, vertex operator algebras, quantum groups and so on - see the recent papers $[15,23,32]$ and the references therein. If we look at Poisson algebras as the 'differential' version of Hopf algebras, then, mutatismutandis, Jacobi algebras can be seen as generalizations of Poisson algebras in the same way as weak Hopf algebras [10] generalize Hopf algebras. It is therefore natural to expect that Jacobi algebras will play an important role in all fields enumerated above. For further details on the study of Jacobi algebras from geometric view point we refer to $[20,22,23,38,45]$.

The paper is organized as follows: in Section 1 we survey the basic concepts that will be used throughout the paper. In particular, we recall the classical concept of a Frobenius algebra together with the corresponding notion for Lie algebras which we will call Frobenius Lie algebra. The latter were previously studied both in mathematics $[28,40]$ and physics $[17,42]$ under the name of self-dual or metric Lie algebras. The property of being Frobenius reflects a certain natural symmetry: for instance, a functor $F: \mathcal{C} \rightarrow \mathcal{D}$ is called Frobenius [13] if $F$ has the same left and right adjoint functor, while a finite dimensional algebra $A$ is called Frobenius [27] if $A \cong A^{*}$, as right $A$-modules, which is the module version of the classical problem of Frobenius asking when two canonical representations of $A$ are equivalent. This idea will be used in Section 2 in the definition of Frobenius Jacobi algebras. For more details on the importance of Frobenius algebras as well as their applications to topology, algebraic geometry and 2D topological quantum field theories we refer to [29], for their categorical generalization at the level of various types of (co)modules 
to $[11,13]$ and for recent contributions and their relevance to other fields (category theory, Hochschild cohomology or graph theory, etc.) see [8, 25, 31] and their references. In Section 2 we present basic properties of Jacobi algebras: in particular, we classify all complex Jacobi algebras of dimension 2 or 3 . The conformal deformation of a Jacobi algebra $A$ (Proposition 2.6) is the Jacobi version of the Drinfel'd twist deformation of a quantum group. We introduce the category $\mathcal{J} \mathcal{M}_{A}^{A}$ of Jacobi A-modules which is an equivalent way of defining representations of a Jacobi algebra $A$ and based on this concept we define a Frobenius Jacobi algebra as a Jacobi algebra $A$ such that $A \cong A^{*}$, as Jacobi $A$-modules. A weaker version of this notion, at the level of Poisson algebras, was recently introduced in [47] where the term Frobenius Poisson algebra is used to denote Poisson algebras $A$ such that $A \cong A^{*}$, isomorphism of right $A$-modules. The concept of integral on a Jacobi algebra is introduced in Definition 2.13 having the Hopf algebra theory as source of inspiration and it is used in the characterization theorem of Frobenius Jacobi algebras (Theorem 2.16): a Jacobi algebra $A$ is Frobenius if and only if there exists a nondegenerate integral on $A$; in the finite dimensional case, this is also equivalent to the existence of a so-called Jacobi-Frobenius pair of $A$, which allows us to define the Euler-Casimir element associated to any finite dimensional Frobenius Jacobi algebra.

The classification of finite dimensional Poisson manifolds is a difficult task: the first steps towards the classification of low dimensional Poisson manifolds were taken in [21,32] using mainly differential geometry tools. It is natural to ask a more general question namely that of classifying Jacobi manifolds of a given dimension. Following the viewpoint of this paper we look at its algebraic counterpart by asking about the classification of all Jacobi algebras of a given dimension. The problem is very difficult since it contains as subsequent questions the classical problems of classifying all associative (resp. Lie) algebras of a given dimension. For updates on the progress made so far and the geometrical significance we refer to [7,39] (resp. [43]) and their list of references - we just mention that the classification of all complex associative (resp. Lie) algebras is known only up to dimension 5 (resp. 7). One of the main tools which was intensively used in the classification of finite 'objects' is the famous extension problem initiated at the level of groups by Hölder and developed later on for Lie algebras, associative algebras, Hopf algebras, Poisson algebras, etc. A more general version of the extension problem is the extending structures problem (ES-problem) introduced in [3] for arbitrary categories. Section 3 is devoted to the study of the ES-problem for Jacobi algebras which consists of the following question:

Let $A$ be a Jacobi algebra and $E$ a vector space containing $A$ as a subspace. Describe and classify the set of all Jacobi algebra structures that can be defined on E such that A becomes a Jacobi subalgebra of $E$.

If we fix $V$ a complement of $A$ in the vector space $E$ then the ES-problem can be rephrased equivalently as follows: describe and classify all Jacobi algebras 
containing $A$ as a subalgebra of codimension equal to $\operatorname{dim}(V)$. The answer to the ES-problem is given in three steps: in the first step (Theorem 3.2) we shall construct the unified product $A \ltimes V$ associated to a Jacobi algebra $A$ and a vector space $V$ connected through four 'actions' and two 'cocycles'. The unified product is a very general construction containing as special cases the semi-direct product, the crossed product or the bicrossed product. The second step (Proposition 3.4) shows that a Jacobi algebra structure $\left(E, *_{E},[-,-]_{E}\right)$ on $E$ contains $A$ as a Jacobi subalgabra if and only if there exists an isomorphism of Jacobi algebras $\left(E, *_{E},[-,-]_{E}\right) \cong A \ltimes V$. Finally, the theoretical answer to the ES-problem is given in Theorem 3.7: for a fixed complement $V$ of $A$ in $E$, a non-abelian cohomological type object $\mathcal{J H}^{2}(V, A)$ is explicitly constructed; it parameterizes and classifies all Jacobi algebras containing $A$ as a subalgebra of codimension equal to $\operatorname{dim}(V)$. The classification obtained in this theorem follows the viewpoint of the extension problem: that is, up to an isomorphism of Jacobi algebras that stabilizes $A$ and co-stabiliezes $V$. For the sake of completeness we also write down the corresponding results for Poisson algebras. Computing the classifying object $\mathcal{J H}^{2}(V, A)$, for a given Jacobi algebra $A$ and a vector space $V$ is a highly nontrivial question. If $A:=k$, the base field viewed as a Jacobi algebra with the trivial bracket, then $\mathcal{J H}^{2}(V, k)$ classifies in fact all Jacobi algebras of dimension $1+\operatorname{dim}(V)$, which is of course a hopeless question for vector spaces of large dimension. For this reason we shall assume from now on that $A \neq k$. A very important step in computing $\mathcal{J H}^{2}(V, A)$ is given in Corollary 3.9, where a decomposition of it as a coproduct over all Jacobi $A$-modules structures on $V$ is given. Section 4 is devoted to computing $\mathcal{J H}^{2}(V, A)$ for what we have called flag Jacobi algebras over $A$ : that is, a Jacobi algebra structure on $E$ such that there exists a finite chain of Jacobi subalgebras $E_{0}:=A \subset E_{1} \subset \cdots \subset E_{m}:=E$, such that each $E_{i}$ has codimension 1 in $E_{i+1}$. All flag Jacobi algebras over $A$ can be completely described by a recursive reasoning where the key step is settled in Theorem 4.6: several applications and examples are given at the end of the section. In particular, if $A$ is a Poisson algebra we indicate the difference between $\mathcal{J H}^{2}(V, A)$ and $\mathcal{P} \mathcal{H}^{2}(V, A)$, the latter being the classifying object of the ES-problem for Poisson algebras.

Section 5 has as starting point the following remark: the necessay and sufficient axioms for the construction of the unified product, applied for (not necessarily unital) Poisson algebras and for trivial cocycles reduces precisely to the definition of the matched pairs of Poisson algebras (Definition 5.1) which were recently introduced by $\mathrm{Ni}$ and Bai [41, Theorem 1] using the equivalent language of representations. Moreover, the associated unified product in this case is precisely the bicrossed product of Poisson algebras. Proposition 5.2 shows that the bicrossed product is the construction responsible for the factorization problem at the level of Poisson algebras. The rest of the section is devoted to the converse of the factorization problem - we call it the bicrossed descent (or the classification of complements) problem $[2,4]$ which for Poisson algebras comes down to the following question: 
Let $P \subset R$ be an extension of Poisson algebras. If a $P$-complement in R exists (i.e. a Poisson subalgebra $Q \subset R$ such that $R=P+Q$ and $P \cap Q=\{0\})$, describe explicitly, classify all $P$-complement in $R$ and compute the cardinal of the isomorphism classes of all $P$-complements in $R$ (which will be called the factorization index $[R: P]^{f}$ of $P$ in $R$ ).

Let $Q$ be a given $P$-complement and $(P, Q, \triangleleft, \triangleright, \angle, \rightarrow)$ the associated canonical matched pair (Proposition 5.2). In Theorem 5.6 a general deformation of the Poisson algebra $Q$ is introduced: it is associated to a deformation map $r: Q \rightarrow P$ in the sense of Definition 5.4. Theorem 5.6 proves that in order to find all complements of $P$ in $R$ it is enough to know only one $P$-complement: all the other $P$-complements are deformations of it. The answer to the bicrossed descent problem is given in Theorem 5.9: there exists a bijection between the isomorphism classes of all $P$-complements of $R$ and a new cohomological type object $\mathcal{H} \mathcal{A}^{2}(P, Q \mid(\triangleleft, \triangleright,\llcorner, \rightarrow))$ which is constructed and the formula for computing the factorization index $[R: P]^{f}$ is given. Examples are also provided: in particular, an extension $P \subseteq R$ of Poisson algebras such that $P$ has infinitely many non-isomorphic complements in $R$ is constructed in Proposition 5.10.

\section{Preliminaries}

For a family of sets $\left(X_{i}\right)_{i \in I}$ we shall denote by $\sqcup_{i \in I} X_{i}$ their coproduct in the category of sets, that is $\sqcup_{i \in I} X_{i}$ is the disjoint union of $X_{i}$, for all $i \in I$. Unless otherwise specified all vector spaces, (bi)linear maps, tensor products are over an arbitrary field $k$. A map $f: V \rightarrow W$ between two vector spaces is called trivial if $f(v)=0$, for all $v \in V . V^{*}=\operatorname{Hom}_{k}(V, k)$ and $\operatorname{End}_{k}(V)$ denote the dual, respectively the endomorphisms ring of a vector space $V$. Throughout we use the following convention: except for the situation when we deal with Poisson algebras as in Section 5 , by an algebra $A=\left(A, m_{A}\right)$ we will always mean an associative, commutative and unital algebra over $k$; the unit of $A$ will be denoted by $1_{A}$ while the multiplication $m_{A}$ is denoted by juxtaposition $m_{A}(a, b)=a b$. All morphisms of algebras preserve units and any left/right $A$-module is unitary. For an algebra $A$ we shall denote by ${ }_{A} \mathcal{M}_{A}$ the category of all $A$-bimodules, i.e. triples $(V, \rightarrow, \triangleleft)$ consisting of a vector space $V$ and two bilinear maps $\rightarrow: A \times V \rightarrow V, \triangleleft: V \times$ $A \rightarrow V$ such that $(V, \rightarrow)$ is a left $A$-module, $(V, \triangleleft)$ is a right $A$-module and $a \rightarrow$ $(x \triangleleft b)=(a \rightarrow x) \triangleleft b$, for all $a, b \in A$ and $x \in V$. Although all algebras considered in this paper are commutative we will maintain the adjectives left/right when defining modules in order to clearly indicate the way actions are defined. For unexplained concepts pertaining to Lie algebra theory we refer to [24]. In particular, representations of a Lie algebra $\mathfrak{g}$ will be viewed as right Lie $\mathfrak{g}$-modules. Explicitly, a right Lie $\mathfrak{g}$-module is a vector space $V$ together with a bilinear map $\angle: V \times \mathfrak{g} \rightarrow V$ 
such that $x<[a, b]=(x<a)<b-(x<b)<a$, for all $a, b \in \mathfrak{g}$ and $x \in V$. Left Lie $\mathfrak{g}$-modules are defined analogously and the category of right Lie $\mathfrak{g}$-modules will be denoted by $\mathcal{L} \mathcal{M}^{\mathfrak{g}}$.

An algebra $A$ is called a Frobenius algebra if $A \cong A^{*}$ as right $A$-modules, where $A^{*}$ is viewed as a right $A$-module via $\left(a^{*} \cdot a\right)(b):=a^{*}(a b)$, for all $a^{*} \in A^{*}$ and $a, b \in A$. For the basic theory of Frobenius algebras we refer to [27]. The Lie algebra counterpart of Frobenius algebras was studied under different names such as self-dual, metric or Lie algebras having a non-degenerate invariant bilinear form. In this paper we will call them Frobenius Lie algebras: a Frobenius Lie algebra is a Lie algebra $\mathfrak{g}$ such that $\mathfrak{g} \cong \mathfrak{g}^{*}$ as right Lie $\mathfrak{g}$-modules, where $\mathfrak{g}$ and $\mathfrak{g}^{*}$ are right Lie $\mathfrak{g}$-modules via the canonical actions: $b<a:=[b, a]$ and $\left(a^{*} \curvearrowleft a\right)(b):=$ $a^{*}([a, b])$, for all $a, b \in \mathfrak{g}$ and $a^{*} \in \mathfrak{g}^{*}$. We can easily see that a Lie algebra $\mathfrak{g}$ is Frobenius if and only if there exists a non-degenerate invariant bilinear form $B: \mathfrak{g} \times \mathfrak{g} \rightarrow k$, i.e. $B([a, b], c)=B(a,[b, c])$, for all $a, b, c \in \mathfrak{g}$. In light of this reformulation, the second Cartan's criterion shows that any finite dimensional complex semisimple Lie algebra is Frobenius since its Killing form is non-degenerate and invariant. Let $\mathfrak{h}(2 n+1, k)$ be the $(2 n+1)$-dimensional Heisenberg algebra: it has a basis $\left\{x_{1}, \cdots, x_{n}, y_{1}, \cdots, y_{n}, z\right\}$ and the only non-zero Lie brackets are $\left[x_{i}, y_{i}\right]:=z$, for all $i=1, \cdots, n$. Then $\mathfrak{h}(2 n+1, k)$ is not Frobenius: if $B$ : $\mathfrak{h}(2 n+1, k) \times \mathfrak{h}(2 n+1, k) \rightarrow k$ is an invariant bilinear form then we can see that $B(z,-)=0$, that is $B$ is degenerate. Besides the mathematical interest in studying Frobenius Lie algebras $[28,40]$, they are also important and have been intensively studied in physics $[17,42]-$ in particular for the construction of Wess-ZuminoNovikov-Witten models.

A Poisson algebra is a triple $A=\left(A, m_{A},[-,-]\right)$, where $\left(A, m_{A}\right)$ is a (not necessarily unital) commutative algebra, $(A,[-,-])$ is a Lie algebra such that the Leibniz law

$$
[a b, c]=a[b, c]+[a, c] b
$$

holds for any $a, b, c \in A$. For further details concerning the study of Poisson algebras arising from differential geometry see [32] and the references therein. If a Poisson algebra $A$ has a unit $1_{A}$, then by taking $a=b=1_{A}$ in the Leibniz law we obtain that $\left[1_{A}, c\right]=\left[c, 1_{A}\right]=0$, for all $c \in A$. Any non unital Poisson algebra embeds into a unital Poisson algebra. If $A$ is a unital Poisson algebra, then using that $\left[a, 1_{A}\right]=0$ and the Jacobi identity, we can easily prove that the map

$$
R: A \otimes A \rightarrow A \otimes A, \quad R(a \otimes b):=b \otimes a+1_{A} \otimes[a, b]
$$

for all $a, b \in A$ is a solution of the quantum Yang-Baxter equation $R^{12} R^{23} R^{12}=$ $R^{23} R^{12} R^{23}$ in $\operatorname{End}_{k}(A \otimes A \otimes A)$. A (right) Poisson $A$-module $[35,47]$ is a vector space $V$ equipped with two bilinear maps $\triangleleft: V \times A \rightarrow V$ and $\angle: V \times A \rightarrow V$ such that $(V, \triangleleft)$ is a right $A$-module, $(V, \sqcup)$ is a right Lie $A$-module satisfying the 
following two compatibility conditions for any $a, b \in A$ and $x \in V$ :

$$
\begin{aligned}
& x \triangleleft(a b)=(x<a) \triangleleft b+(x<b) \triangleleft a, \\
& x \triangleleft[a, b]=(x \triangleleft a)<b-(x<b) \triangleleft a
\end{aligned}
$$

We denote by $\mathcal{P} \mathcal{M}_{A}^{A}$ the category of right Poisson $A$-modules having as morphisms all linear maps which are compatible with both actions.

Unified products for associative/Lie algebras. We recall some concepts and constructions from $[5,7]$ that will be used from Section 3 on.

Definition 1.1. Let $A$ be an algebra and $V$ a vector space. An algebra extending system of $A$ through $V$ is a system $\Omega(A, V)=(\triangleleft, \triangleright, f, \cdot)$ consisting of four bilinear maps $\triangleleft: V \times A \rightarrow V, \triangleright: V \times A \rightarrow A, \quad f: V \times V \rightarrow A, \quad \cdot: V \times V \rightarrow V$ satisfying the following six compatibility conditions for any $a, b \in A, x, y, z \in V$ :

(A1) $f$ and $\cdot$ are symmetric, $(V, \triangleleft)$ is a right $A$-module and $x \triangleright 1_{A}=0$

(A2) $x \cdot(y \cdot z)-(x \cdot y) \cdot z=z \triangleleft f(x, y)-x \triangleleft f(y, z)$

(A3) $(x \cdot y) \triangleleft a=x \triangleleft(y \triangleright a)+x \cdot(y \triangleleft a)$

(A4) $x \triangleright(a b)=a(x \triangleright b)+(x \triangleleft b) \triangleright a$

(A5) $(x \cdot y) \triangleright a=x \triangleright(y \triangleright a)+f(x, y \triangleleft a)-f(x, y) a$

(A6) $f(x, y \cdot z)-f(x \cdot y, z)=z \triangleright f(x, y)-x \triangleright f(y, z)$

Let $\Omega(A, V)=(\triangleleft, \triangleright, f, \cdot)$ be an extending system of $A$ through $V$ and $A \ltimes_{\Omega(A, V)} V:=A \times V$ with the multiplication $\bullet$ defined for any $a, b \in A$ and $x$, $y \in V$ by:

$$
(a, x) \bullet(b, y):=(a b+x \triangleright b+y \triangleright a+f(x, y), x \triangleleft b+y \triangleleft a+x \cdot y)
$$

Then $A \ltimes_{\Omega(A, V)} V=\left(A \ltimes_{\Omega(A, V)} V, \bullet\right)$ is a commutative algebra having $\left(1_{A}, 0_{V}\right)$ as a unit, called the unified product of $A$ and $\Omega(A, V)$. In fact, there is more to be said: $\left(A \ltimes_{\Omega(A, V)} V, \bullet\right)$ is a commutative algebra with the unit $\left(1_{A}, 0_{V}\right)$ if and only if $\Omega(A, V)$ is an extending system of $A$ through $V$ - this is the commutative version of [7, Theorem 2.2]. Any unified product $A \ltimes_{\Omega(A, V)} V$ contains $A \cong A \times\{0\}$ as a subalgebra. Conversely, let $A$ be an algebra and $E$ a vector space containing $A$ as a subspace. Then, any algebra structure - on $E$ containing $A$ as a subalgebra is isomorphic to a unified product, that is $(E, \cdot) \cong A \ltimes_{\Omega(A, V)} V$, for some extending system of $A$ through $V$ - this is [7, Theorem 2.4] applied for the special case of commutative algebras. 
Example 1.2. Let $A$ be an algebra. Then there is a bijection between the set of all algebra extending systems of $A$ through $k$ and the set of all 4-tuples $\left(\Lambda, \Delta, f_{0}, u\right) \in$ $A^{*} \times \operatorname{End}_{k}(A) \times A \times k$ satisfying the following compatibilities for any $a, b \in A$ :

(FA1) $\Lambda: A \rightarrow k$ is an algebra map and $\Lambda \circ \Delta=0$

(FA2) $\Delta(a b)=a \Delta(b)+\Lambda(b) \Delta(a)$

(FA3) $\Delta^{2}(a)=u \Delta(a)+f_{0} a-\Lambda(a) f_{0}$

The bijection is given such that the algebra extending system $\Omega(A, k)=(\triangleleft, \triangleright, f, \cdot)$ associated to $\left(\Lambda, \Delta, f_{0}, u\right)$ is defined for any $x, y \in k$ and $a \in A$ by:

$$
x \triangleleft a:=x \Lambda(a), \quad x \triangleright a:=x \Delta(a), \quad f(x, y):=x y f_{0}, \quad x \cdot y:=x y u
$$

A 4-tuple $\left(\Lambda, \Delta, f_{0}, u\right)$ satisfying (FA1)-(FA3) is called a flag datum of $A$ and we denote by $\mathcal{F}(A)$ the set of all flag datums of $A$. The unified product $A \ltimes_{\Omega(A, k)} k$ associated to a flag datum $\left(\Lambda, \Delta, f_{0}, u\right)$ will be denoted by $A_{\left(\Lambda, \Delta, f_{0}, u\right)}$ and coincides with the vector space $A \times k$ having the multiplication given for any $a$, $b \in A, x, y \in k$ by:

$$
(a, x) \bullet(b, y):=\left(a b+x \Delta(b)+y \Delta(a)+x y f_{0}, x \Lambda(b)+y \Lambda(a)+x y u\right)
$$

An algebra $B$ contains $A$ as a subalgebra of codimension 1 if and only if $B \cong$ $A_{\left(\Lambda, \Delta, f_{0}, u\right)}$, for some flag datum $\left(\Lambda, \Delta, f_{0}, u\right) \in \mathcal{F}(A)$ [7, Section 4].

The Lie algebra counterpart of the extending structures were introduced in [5] as follows:

Definition 1.3. Let $A=(A,[-,-])$ be a Lie algebra and $V$ a vector space. A Lie extending system of $A$ through $V$ is a system $\Lambda(A, V)=(<, \rightarrow, \theta,\{-,-\})$ consisting of four bilinear maps $\leftarrow: V \times A \rightarrow V, \rightarrow: V \times A \rightarrow A, \theta: V \times V \rightarrow A$, $\{-,-\}: V \times V \rightarrow V$ satisfying the following compatibility conditions for any $a$, $b \in A, x, y, z \in V$ :

(L1) $(V, \leftarrow)$ is a right Lie $A$-module, $\theta(x, x)=0$ and $\{x, x\}=0$

(L2) $x \rightarrow[a, b]=[x \rightarrow a, b]+[a, x \rightarrow b]+(x<a) \rightarrow b-(x<b) \rightarrow a$

(L3) $\{x, y\}<a=\{x, y<a\}+\{x<a, y\}+x<(y \rightarrow a)-y<(x \rightarrow a)$

(L4) $\{x, y\} \rightarrow a=x \rightarrow(y \rightarrow a)-y \rightarrow(x \rightarrow a)+[a, \theta(x, y)]$

$$
+\theta(x, y<a)+\theta(x<a, y)
$$

(L5) $\sum_{(c)} \theta(x,\{y, z\})+\sum_{(c)} x \rightarrow \theta(y, z)=0$

(L6) $\sum_{(c)}\{x,\{y, z\}\}+\sum_{(c)} x<\theta(y, z)=0$

where $\sum_{(c)}$ denotes the circular sum. 
The concept of extending system of a Lie algebra $A$ through a vector space $V$ generalizes the concept of a matched pair of Lie algebras as defined in [36,37]: if $\theta$ is the trivial map, then $\Lambda(A, V)=(\llcorner, \rightarrow, \theta:=0,\{-,-\})$ is a Lie extending system of $A$ through $V$ if and only if $(V,\{-,-\})$ is a Lie algebra and $(A, V, \leftarrow, \rightarrow)$ is a matched pair of Lie algebras. Let $\Lambda(A, V)=(\llcorner, \rightarrow, \theta,\{-,-\})$ be an extending system of a Lie algebra $A$ through a vector space $V$ and let $A \ltimes_{\Lambda(A, V)} V$ be the vector space $A \times V$ with the bracket [-, - ] defined for any $a, b \in A$ and $x, y \in V$ by:

$[(a, x),(b, y)]:=([a, b]+x \rightarrow b-y \rightarrow a+\theta(x, y),\{x, y\}+x<b-y<a)$

Then $A \ltimes_{\Lambda(A, V)} V$ is a Lie algebra called the unified product of $A$ and $\Lambda(A, V)$. Moreover, [5, Theorem 2.2] proves that $\left(A \ltimes_{\Lambda(A, V)} V,[-,-]\right)$ with the bracket given by (1.4) is a Lie algebra if and only if $\Lambda(A, V)$ is a Lie extending system of $A$ through $V$. The Lie algebra $A \ltimes_{\Lambda(A, V)} V$ contains $A \cong A \times\{0\}$ as a Lie subalgebra. Conversely, let $A$ be a Lie algebra, $E$ a vector space such that $A$ is a subspace of $E$. Then, any Lie algebra structure [-, - ] on $E$ containing $A$ as a Lie subalgebra is isomorphic to a unified product: i.e., $(E,[-,-]) \cong A \ltimes_{\Lambda(A, V)} V$, for some $\Lambda(A, V)=(\leftarrow, \rightarrow, \theta,\{-,-\})$, an extending system of $A$ through $V$ ([5, Theorem 2.4]).

Example 1.4. Let $A=(A,[-,-])$ be a Lie algebra. [5, Proposition 4.4] proves that there is a bijection between the set of all Lie extending system of $A$ through $k$ and the set $\operatorname{TwDer}(A)$ of all twisted derivations of $A$, which is the set of all pairs $(\lambda, D) \in A^{*} \times \operatorname{End}_{k}(A)$ satisfying the following compatibilities for any $a, b \in A$ :

(FL1) $\lambda([a, b])=0$

(FL2) $D([a, b])=[D(a), b]+[a, D(b)]+\lambda(a) D(b)-\lambda(b) D(a)$

The bijection is given such that the Lie extending system $\Lambda(A, k)=(\llcorner, \rightarrow$, $\theta,\{-,-\})$ associated to a twisted derivation $(\lambda, D)$ is defined for any $x, y \in k$ and $a \in A$ by:

$$
x<a:=x \lambda(a), \quad x \rightarrow a:=x D(a), \quad \theta(x, y):=0, \quad\{x, y\}:=0
$$

$\operatorname{TwDer}(A)$ contains the usual space of derivations $\operatorname{Der}(A)$ via the canonical embedding $\operatorname{Der}(A) \hookrightarrow \operatorname{Tw} \operatorname{Der}(A), D \mapsto(0, D)$. We point out that the above canonical embedding is bijective if $A$ is a perfect Lie algebra. The unified product $A \ltimes_{\Lambda(A, k)} k$ associated to $(\lambda, D) \in \operatorname{TwDer}(A)$ is denoted by $A_{(\lambda, D)}$ and it is the vector space $A \times k$ with the bracket defined for any $a, b \in A$ and $x, y \in k$ by:

$$
[(a, x),(b, y)]:=([a, b]+x D(b)-y D(a), x \lambda(b)-y \lambda(a))
$$

A Lie algebra $\mathfrak{g}$ contains $A$ as a Lie subalgebra of codimension 1 if and only if $\mathfrak{g} \cong A_{(\lambda, D)}$, for some $(\lambda, D) \in \operatorname{Tw} \operatorname{Der}(A)[5$, Section 4$]$. 


\section{Basic concepts, (bi)modules, integrals and Frobenius Jacobi algebras}

We recall the definition of Jacobi algebras $[20,38]$ as the abstract algebraic counterpart of Jacobi manifolds:

Definition 2.1. A Jacobi algebra is a quadruple $A=\left(A, m_{A}, 1_{A},[-,-]\right)$, where $\left(A, m_{A}, 1_{A}\right)$ is a unital algebra, $(A,[-,-])$ is a Lie algebra such that for any $a, b$, $c \in A$ :

$$
[a b, c]=a[b, c]+[a, c] b-a b\left[1_{A}, c\right]
$$

Any unital Poisson algebra is a Jacobi algebra. Any algebra $A$ is a Jacobi algebra with the trivial bracket $[a, b]=0$, for all $a, b \in A$ - such an Jacobi algebra will be called abelian and will be denoted by $A_{0}$. A morphism between two Jacobi (resp. Poisson) algebras $A$ and $B$ is a linear map $\varphi: A \rightarrow B$ which is both a morphism of algebras as well as a morphism of Lie algebras. We denote by ${ }_{k} \mathrm{Jac}$ (resp. ${ }_{k}$ Poss) the category of Jacobi (resp. unitary Poisson) algebras over a field $k$. A Jacobi ideal of a Jacobi algebra $A$ is a linear subspace $I$ which is both an ideal with respect to the associative product as well as a Lie ideal of $A$. If $I$ is a Jacobi ideal of $A$ then $A / I$ inherits a Jacobi algebra structure in the obvious way.

Remarks 2.2. (1) The category ${ }_{k}$ Poss of unital Poisson algebras is a reflective subcategory in the category of Jacobi algebras, i.e. the inclusion functor $\iota:{ }_{k}$ Poss $\hookrightarrow$ ${ }_{k}$ Jac has a left adjoint which we will denote by $(-)_{\text {poss }}:{ }_{k}$ Jac $\rightarrow{ }_{k}$ Poss and whose construction goes as follows: for any Jacobi algebra $A$ we define $A_{\text {poss }}:=A / I_{\text {poss }}$, where $I_{\text {poss }}$ is the Jacobi ideal of $A$ generated by all brackets $\left[1_{A}, a\right]$, for all $a \in A$. Then, $A_{\text {poss }}$ is a Poisson algebra and the quotient map $A \rightarrow A_{\text {poss }}, a \mapsto \widehat{a}$ is universal among the maps from $A$ to any unital Poisson algebra which are morphisms of Jacobi algebras. We mention that it is possible that $A_{\text {poss }}=0$ - this happens when $I_{\text {poss }}$ contains an invertible element of $A$. An example is the Jacobi algebra $J^{2,2}$ defined in Example 2.3 below.

(2) The category ${ }_{k} \mathrm{Jac}=\left({ }_{k} \mathrm{Jac},-\otimes-, k, \tau_{-,-}\right)$is braided monoidal: if $A$ and $B$ are Jacobi algebras, then $A \otimes B$ is a Jacobi algebra via

$(a \otimes b) \cdot\left(a^{\prime} \otimes b^{\prime}\right):=a a^{\prime} \otimes b b^{\prime}, \quad\left[a \otimes b, a^{\prime} \otimes b^{\prime}\right]:=a a^{\prime} \otimes\left[b, b^{\prime}\right]+\left[a, a^{\prime}\right] \otimes b b^{\prime}$

for all $a, a^{\prime} \in A$ and $b, b^{\prime} \in B$, the base field $k$ viewed with the abelian Lie bracket is the unit and the braiding is the usual flip $\tau_{A, B}: A \otimes B \rightarrow B \otimes A$, $\tau_{A, B}(a \otimes b):=b \otimes a$.

The classification of Jacobi algebras of a given finite dimension is the first nontrivial question which arises as the algebraic counterpart of the classification of finite dimensional Jacobi manifolds. Of course, any 1-dimensional Jacobi algebra is isomorphic to the abelian Jacobi algebra $k_{0}$. The 2-dimensional case is covered below and it reveals an interesting fact namely that two Jacobi algebras can be isomorphic both as algebras and Lie algebras (through different isomorphisms) but not isomorphic as Jacobi algebras. A similar result holds for Hopf algebras as well. 
Example 2.3. Let $k$ be a field of characteristic $\neq 2$. If $k=k^{2}:=\left\{q^{2} \mid q \in k\right\}$ then, up to an isomorphism, there exist four 2-dimensional Jacobi algebras over $k$. These are the Jacobi algebras denoted by $J^{2,1}, J^{2,2}, J^{2,3}, J^{2,4}$ having $\{1, x\}$ as a basis and the multiplication and the bracket defined by:

$$
\begin{array}{llll}
J^{2,1}: x^{2}=0, & {[1, x]=0 ;} & J^{2,2}: x^{2}=0, & {[1, x]=1} \\
J^{2,3}: x^{2}=x, & {[1, x]=0 ;} & J^{2,4}: x^{2}=0, & {[x, 1]=x}
\end{array}
$$

If $k \neq k^{2}$, besides the four Jacobi algebras listed above there exists another oneparameter 2-dimensional Jacobi algebra $J_{d}^{2}$ defined by:

$$
J_{d}^{2}: x^{2}=d, \quad[1, x]=0
$$

for all $d \in S$, where $S \subseteq k \backslash k^{2}$ a system of representatives for the following equivalence relation on $k \backslash k^{2}: d \equiv d^{\prime}$ if and only if there exists $q \in k^{*}$ such that $d=q^{2} d^{\prime}$.

Indeed, we will fix $\{1, x\}$ as a basis in a two dimensional Jacobi algebra. The proof follows from the classical classification of 2-dimensional Lie algebras [34] and from the well known classification of 2-dimensional associative algebras [46] (for arbitrary fields see [7, Corollary 4.5]). Indeed, the classification follows by a routine computation based on checking the compatibility condition (2.1). We only mention that the algebra defined by the multiplication $x^{2}=x$ (or $x^{2}=d$, for some $d \in S$, if $k \neq k^{2}$ ) together with the Lie bracket $[1, x]=1$ or $[1, x]=x$ is not a Jacobi algebra since the compatibility condition (2.1) fails for $a=b=c:=x$. We observe that $J^{2,2}$ and $J^{2,4}$ are Jacobi non-Poisson algebras, $\left(J^{2,2}\right)_{\text {poss }}=0$ and $\left(J^{2,4}\right)_{\text {poss }} \cong k_{0}$. Moreover, the Jacobi algebras $J^{2,2}$ and $J^{2,4}$ are isomorphic as associative algebras as well as Lie algebras but they are not isomorphic as Jacobi algebras.

In particular, if $k=\mathbb{C}$ there are four isomorphism classes of 2-dimensional Jacobi algebras, while if $k=\mathbb{R}$ there exist five types of 2-dimensional Jacobi algebras, the fifth one being the Jacobi algebra $J_{-1}^{2}$.

Example 2.4. Using the classical classification of 3-dimensional associative (resp. Lie) algebras from [46] (resp. [26,34]) over the complex field $\mathbb{C}$ and the same strategy as in Example 2.3 we can prove that, up to an isomorphism, there are exactly 11 families of complex Jacobi algebras of dimension 3: they have $\{1, x, y\}$ as a basis and the multiplication and the bracket as listed in Table 1.

Subsequent to the problem of classifying Jacobi algebras of a given dimension is the following question: for a given algebra (resp. Lie algebra) A, describe and classify all Lie brackets $[-,-]$ (resp. all possible multiplications $m_{A}$ ) which endow $A$ with a Jacobi algebra structure. Some examples are given below: 


\begin{tabular}{|l|c|c|}
\hline Jacobi algebra & Algebra structure & Lie bracket \\
\hline$J_{1}^{3}$ & $x^{2}=y^{2}=x y=y x=0$ & abelian \\
\hline$J_{2}^{3}$ & $x^{2}=y, y^{2}=0, x y=y x=0$ & abelian \\
\hline$J_{3}^{3}$ & $x^{2}=x, y^{2}=0, x y=y x=0$ & abelian \\
\hline$J_{4}^{3}$ & $x^{2}=x, y^{2}=y, x y=y x=0$ & abelian \\
\hline$J_{5}^{3}$ & $x^{2}=y^{2}=x y=y x=0$ & {$[\mathrm{x}, 1]=\mathrm{x}$} \\
\hline$J_{6}^{3}$ & $x^{2}=y^{2}=x y=y x=0$ & {$[\mathrm{x}, \mathrm{y}]=\mathrm{x}$} \\
\hline$J_{7}^{3}$ & $x^{2}=y^{2}=x y=y x=0$ & {$[\mathrm{x}, 1]=\mathrm{x}+\mathrm{y},[\mathrm{y}, 1]=\mathrm{y}$} \\
\hline${ }^{3} J_{8}^{3}, u \in k^{*}$ & $x^{2}=y^{2}=x y=y x=0$ & {$[\mathrm{x}, 1]=\mathrm{x},[\mathrm{y}, 1]=\mathrm{y}$} \\
\hline$J_{9}^{3}$ & $x^{2}=y, y^{2}=0, x y=y x=0$ & {$[\mathrm{x}, 1]=\mathrm{x},[\mathrm{y}, 1]=2 \mathrm{y}$} \\
\hline$J_{10}^{3}$ & $x^{2}=y, y^{2}=0, x y=y x=0$ & {$[\mathrm{x}, 1]=2^{-1} \mathrm{x},[\mathrm{y}, 1]=\mathrm{y}$} \\
\hline$J_{11}^{3}$ & $x^{2}=x, y^{2}=0, x y=y x=0$ & {$[\mathrm{y}, 1]=\mathrm{y}$} \\
\hline
\end{tabular}

Table 1. Jacobi algebras of dimension 3 over $\mathbb{C}$.

Examples 2.5. (1) Let $C_{n}$ be a cyclic group of order $n \geq 2$ generated by $c$. Then the group algebra $k\left[C_{n}\right]$ can be made into a Jacobi algebra as follows:

(1) If $\operatorname{char}(k)=0$ or $\operatorname{char}(k)=p>0$ and $(p, n-1)=1$, then the only Lie bracket which makes $k\left[C_{n}\right]$ into a Jacobi algebra is the trivial one, i.e. $k\left[C_{n}\right]=k\left[C_{n}\right]_{0}$;

(2) If char $(k)=p \mid n-1$, then any $y \in k\left[C_{n}\right]$ induces a unique Lie bracket given by $\left[c^{i}, c^{j}\right]=(j-i) c^{j+i-1} y$, for all $i, j=1,2, \ldots, n$ which makes $k\left[C_{n}\right]$ into a Jacobi algebra.

Indeed, let $[-,-]$ be a Lie bracket that endows the group algebra $k\left[C_{n}\right]$ with a Jacobi algebra structure and we denote $y:=[1, c]$. By using (2.1) and the induction we obtain that $\left[c^{i}, c^{j}\right]=(j-i) c^{j+i-1} y$, for all $i, j=1,2, \ldots, n$. Therefore, since $\left[c^{n}, c\right]=y$ we obtain $(n-1) y=0$, which brings us to the two cases considered above. If char $(k)=p$ and $p \mid n-1$, then it can be easily seen that Jacobi's identity is also fulfilled.

(2) Let $C_{\infty}$ be the infinite cyclic group generated by $c$. Then, for any $y \in k\left[C_{\infty}\right]$, the group algebra $k\left[C_{\infty}\right]$ admits a Jacobi algebra structure with the bracket given by $\left[c^{i}, c^{j}\right]=(j-i) c^{j+i-1} y$, for all $i, j \in \mathbb{Z}$.

(3) Let $\mathrm{sl}(2, \mathbb{C})$ be the complex special linear algebra of dimension 3. Since $\mathrm{sl}(2, \mathbb{C})$ is perfect as a Lie algebra, a careful analysis of the Lie brackets on 3-dimensional Jacobi algebras given in Table 1 brings us to the conclusion that the Lie algebra sl $(2, \mathbb{C})$ can not be endowed with an algebra structure to make it into a Jacobi agebra.

The following construction is the algebraic counterpart of conformal deformations of Jacobi manifolds [38]. Mutatis-mutandis it can be seen as the Jacobi version of the Drinfel'd twist deformation for quantum groups [16]. 
Proposition 2.6. Let $A=\left(A, m_{A},[-,-]\right)$ be a Jacobi algebra and $u \in U(A)$ an invertible element of $A$. Then $A_{u}:=\left(A, m_{A},[-,-]_{u}\right)$ is a Jacobi algebra called the $u$-conformal deformation of $A$, where the bracket $[-,-]_{u}$ is given for any $x, y \in A$ by:

$$
[x, y]_{u}:=u^{-1}[u x, u y]
$$

Proof. The bilinear map $[-,-]_{u}$ is a Lie bracket on $A$ since for any $x, y, z \in A$ we have:

$$
\begin{aligned}
& {\left[x,[y, z]_{u}\right]_{u}+\left[y,[z, x]_{u}\right]_{u}+\left[z,[x, y]_{u}\right]_{u}} \\
& \quad=u^{-1}[u x,[u y, u z]]+u^{-1}[u y,[u z, u x]]+u^{-1}[u z,[u x, u y]]=0
\end{aligned}
$$

Now, the compatibility condition (2.1) is equivalent to $[u x y, u z]=[u x, u z] y+$ $x[u y, u z]-x y[u, u z]$, for all $x, y, z \in A$. The right hand side gives:

$$
\begin{aligned}
& {[u x, u z] y+x \underline{[u y, u z]}-x y[u, u z]} \\
& \quad \stackrel{(2.1)}{=}[u x, u z] y+x\left(u[y, u z]+[u, u z] y-u y\left[1_{A}, u z\right]\right)-x y[u, u z] \\
& \quad=[u x, u z] y+u x[y, u z]-u x y\left[1_{A}, u z\right] \\
& \quad \stackrel{(2.1)}{=}[u x y, u z]
\end{aligned}
$$

as needed, where the last equality follows by applying (2.1) for the bracket $[-,-]$ in $u x, y$ and respectively $u z$.

Describing and classifying all $u$-conformal deformations of a given Jacobi algebra $A$ is an interesting question that will be addressed somewhere else. In what follows we provide an example which shows that a $u$-conformal deformation of a Jacobi algebra can be a Poisson algebra.

Example 2.7. Let $A$ be the 3-dimensional Jacobi non-Poisson algebra over of field of characteristic $\neq 2$ having $\{1, x, y\}$ as a basis and the multiplication and the nontrivial brackets given by $x^{2}:=0, \quad x y=y x:=x, \quad y^{2}:=2 y-1, \quad[1, y]=$ $[x, y]:=x\left(A\right.$ is the Jacobi algebra denoted by $J_{2,0,-1,-1}^{3,5}$ in Proposition 4.10 below). The group $U(A)$ of units of $A$ coincides with the set of elements of the form $u=\alpha+\beta x+\gamma y$, with $\alpha+\gamma \neq 0$ and the space of all $u$-conformal deformations of $A$ is in bijection with the three-parameter Jacobi algebras having the bracket defined by:

$$
[1, x]_{u}=-\gamma x, \quad[1, y]_{u}=(\alpha+\beta+\gamma) x, \quad[x, y]_{u}=(\alpha+2 \gamma) x
$$

for all $\alpha, \beta, \gamma \in k$ such that $\alpha+\gamma \neq 0$. In particular, the $u$-conformal deformation of $A$ corresponding to $u:=\alpha(1-x)$, for any $\alpha \in k^{*}$ is a Poisson algebra. 
(Bi)Modules, integrals and Frobenius Jacobi algebras. We shall introduce the representations of a Jacobi algebra using the equivalent notion of modules.

Definition 2.8. Let $A$ be a Jacobi algebra. A right Jacobi A-module is a vector space $V$ equipped with two bilinear maps $\triangleleft: V \times A \rightarrow V$ and $\measuredangle: V \times A \rightarrow V$ such that $(V, \triangleleft)$ is a right $A$-module, $(V, \triangleleft)$ is a right Lie $A$-module satisfying the following two compatibility conditions for any $a, b \in A$ and $x \in V$ :

$$
\begin{aligned}
& x \triangleleft(a b)=(x<a) \triangleleft b+(x<b) \triangleleft a-\left(x<1_{A}\right) \triangleleft(a b) \\
& x \triangleleft[a, b]=(x \triangleleft a)<b-(x<b) \triangleleft a+(x \triangleleft a) \triangleleft\left[1_{A}, b\right]
\end{aligned}
$$

A right Jacobi $A$-bimodule is a right Jacobi $A$-module which in addition satisfies the following compatibility condition for any $a, b \in A$ and $x \in V$ :

$$
x<(a b)=(x \triangleleft a)<b+(x \triangleleft b)<a-(x \triangleleft(a b))<1_{A}
$$

Let $\mathcal{J M}_{A}^{A}$ (resp. $\mathcal{J B}_{A}^{A}$ ) be the category of right Jacobi $A$-modules (resp. Jacobi $A$-bimodules) having as morphisms the linear maps which are compatible with both actions.

The categories ${ }_{A}^{A} \mathcal{J} \mathcal{M}$ (resp. ${ }_{A}^{A} \mathcal{J} \mathcal{B}$ ) of left Jacobi $A$-(bi)modules are defined analogously and there exists an isomorphism of categories ${ }_{A}^{A} \mathcal{J} \mathcal{M} \cong \mathcal{J} \mathcal{M}_{A}^{A}$ and ${ }_{A}^{A} \mathcal{J} \mathcal{B} \cong \mathcal{J B}_{A}^{A}$.

Remarks 2.9. (1) The compatibility conditions (2.3)-(2.4) defining the category $\mathcal{J M}_{A}^{A}$ are the Jacobi version of the compatibility conditions (1.1) defining Poisson modules over a Poisson algebra: they are precisely axioms (J2) and (J4) from the construction of the unified product (Theorem 3.2). On the other hand, axiom (2.5) defining Jacobi bimodules has another explanation which will be given in Lemma 2.11 below.

(2) Rephrasing Definition 2.8 in terms of representations can be done as follows: a representation of a Jacobi algebra $A$ on a vector space $V$ is a pair $(\psi, \varphi)$ consisting of a representation $\psi$ of the associative algebra $A$ on $V$, that is an algebra map $\psi: A \rightarrow \operatorname{End}_{k}(A)$ and a representation $\varphi$ of a Lie algebra $A$ on $V$, i.e. a Lie algebra map $\varphi: A \rightarrow \operatorname{gl}(V)$, satisfying the following two compatibilities for any $a, b \in A$ :

$$
\begin{aligned}
\varphi(a b) & =\psi(b) \circ \varphi(a)-\psi(a) \circ \varphi(b)+\psi(a b) \circ \varphi\left(1_{A}\right) \\
\psi([a, b]) & =\psi(a) \circ \varphi(b)-\varphi(b) \circ \psi(a)+\psi\left(\left[1_{A}, b\right] a\right)
\end{aligned}
$$

Representations of a Jacobi algebra $A$ and right Jacobi $A$-modules are two different ways of describing the same structure: more precisely, there exists an equivalence of categories $\mathcal{J} \mathcal{M}_{A}^{A} \cong \operatorname{Rep}(A)$, where $\operatorname{Rep}(A)$ is the category of representations of $A$ with the obvious morphisms. The one-to-one correspondence between right Jacobi $A$-modules $(\triangleleft, \measuredangle)$ on $V$ and representations $(\psi, \varphi)$ of $A$ on $V$ is given by the two-sided formulas: $\psi(a)(x):=: x \triangleleft a$ and $\varphi(a)(x):=:-x<a$, for all $a \in A$ and $x \in V$. 
Examples 2.10. (1) Any Jacobi algebra $A$ has a canonical structure of a Jacobi $A$-bimodule via: $x \triangleleft a:=x a$ and $x<a:=[x, a]$, for all $x, a \in A$. Indeed, for these actions, axioms (2.3) and (2.4) are both equivalent to the compatibility condition (2.1) defining a Jacobi algebra. On the other hand, axiom (2.5) is equivalent to

$$
[x, a b]=[x a, b]+[x b, a]-\left[x a b, 1_{A}\right]
$$

which follows trivially by applying several times the compatibility condition (2.1).

(2) Any vector space $V$ can be viewed as a Jacobi $A$-bimodule via the trivial actions: $x \triangleleft a:=x$ and $x<a:=0$, for all $x \in V, a \in A$. We shall denote by $V_{0}$ the vector space $V$ equipped with these trivial actions.

(3) There exist a bijection between the set of all right Jacobi $A$-module structures $(\triangleleft, \angle)$ that can be defined on $k$ and the set of all pairs $(\Lambda, \lambda) \in A^{*} \times A^{*}$, consisting of an algebra map $\Lambda: A \rightarrow k$ and a Lie algebra map $\lambda: A \rightarrow k$ satisfying the following two compatibility conditions for any $a, b \in A$ :

$$
\begin{aligned}
\lambda(a b) & =\lambda(a) \Lambda(b)+\lambda(b) \Lambda(a)-\lambda\left(1_{A}\right) \Lambda(a b), \\
\Lambda([a, b]) & =\Lambda(a) \Lambda\left(\left[1_{A}, b\right]\right)
\end{aligned}
$$

The bijection is given such that the Jacobi $A$-module structure $(\triangleleft, \measuredangle)$ on $k$ associated to $(\Lambda, \lambda) \in A^{*} \times A^{*}$ is defined by $x \triangleleft a:=x \Lambda(a)$ and $x<a:=x \lambda(a)$, for all $a \in A, x \in k$. The actions associated to such a pair $(\Lambda, \lambda)$ endow $k$ with a Jacobi $A$-bimodule structure since (2.5) also holds thanks to the first compatibility condition of (2.8).

The proof of the next lemma provides a motivation for introducing axiom (2.5) in Definition 2.8: without it the linear dual of a right Jacobi $A$-module is not necessarily a right Jacobi $A$-module.

Lemma 2.11. Let $A$ be a Jacobi algebra and $(V, \triangleleft, \sqcup) \in \mathcal{J B}_{A}^{A}$ a Jacobi A-bimodule. Then the $k$-linear dual $V^{*}=\left(V^{*}, \boldsymbol{4}, \curvearrowleft\right) \in \mathcal{J B}_{A}^{A}$ is a Jacobi $A$-bimodule, where the actions $\mathbf{4} \curvearrowleft \curvearrowleft$ are defined for any $v^{*} \in V^{*}, a \in A$ and $x \in V$ by:

$$
\left(v^{*} \triangleleft a\right)(x):=v^{*}(x \triangleleft a), \quad\left(v^{*} \curvearrowleft a\right)(x):=-v^{*}(x<a)
$$

In particular, there exists a well defined contravariant functor $(-)^{*}: \mathcal{J B}_{A}^{A} \rightarrow \mathcal{J B}_{A}^{A}$ which for finite dimensional Jacobi A-bimodules provides a duality of categories.

Proof. $\left(V^{*}, \curvearrowleft\right)$ is a right Lie $A$-module and $\left(V^{*}, 4\right)$ is a right $A$-module since $A$ is a commutative algebra. A straightforward computation shows that the compatibility condition (2.3) holds for $\left(V^{*}, \triangleleft, \curvearrowleft\right)$ if and only if (2.5) holds for $(V, \triangleleft, \hookrightarrow)$ and similar (2.5) holds for $\left(V^{*}, \triangleleft, \curvearrowleft\right)$ if and only if (2.3) holds for $(V, \triangleleft, \measuredangle)$. Finally, (2.4) for $\left(V^{*}, \triangleleft, \curvearrowleft\right)$ is equivalent to (2.4) for $(V, \triangleleft, \measuredangle)$. 
We shall view the Jacobi algebra $A$ as an object in $\mathcal{J B}_{A}^{A}$ via the actions $x \triangleleft a:=x a$ and $x<a:=[x, a]$, for all $x, a \in A$. It follows from Lemma 2.11 that the $k$-linear dual $A^{*}$ is a Jacobi $A$-bimodule whose actions take the following form:

$$
\left(a^{*} \triangleleft a\right)(x):=a^{*}(a x), \quad\left(a^{*} \curvearrowleft a\right)(x):=a^{*}([a, x])
$$

From now on we will see $A$ and $A^{*}$ as objects in $\mathcal{J B}_{A}^{A}$ via the above structures.

Definition 2.12. A finite dimensional Jacobi algebra $A$ is called Frobenius if there exists an isomorphism $A \cong A^{*}$ in $\mathcal{J B}_{A}^{A}$, i.e. an isomorphism of right $A$-modules and right Lie $A$-modules.

Any Frobenius Jacobi algebra is Frobenius as an associative algebra and as a Lie algebra. Now we introduce the concept of integral on a Jacobi algebra: as in the case of Hopf algebras [44], integrals will be intimately related to the property of being Frobenius.

Definition 2.13. An integral on a Jacobi algebra $A$ is an element $v \in A^{*}$ such that

$$
v([a, b] c)=v(a[b, c])
$$

for all $a, b, c \in A$. We denote by $\int_{A}$ the space of integrals on a Jacobi algebra $A$. An integral $v$ is called non-degenerate if: $v(a x)=0$, for all $x \in A$ implies $a=0$.

If $v$ is an integral on $A$ then by taking $b=c=1_{A}$ in (2.11) we obtain that $v([a, 1])=0$.

Examples 2.14. (1) If $A$ is an abelian Jacobi algebra, then $\int_{A}=A^{*}$.

(2) Let $A$ be a unital Poisson algebra and $v \in \int_{A}$. Then by taking $c=1_{A}$ in (2.11) we obtain that $v(x)=0$, for any $x \in A^{\prime}:=[A, A]$. In particular, it follows that $\int_{A}=0$, for any Poisson algebra $A$ which is perfect as a Lie algebra.

(3) The trace map $\operatorname{Tr}: \mathrm{M}_{n}(k) \rightarrow k$ satisfies (2.11) since $\operatorname{Tr}([A, B] C)=$ $\operatorname{Tr}(A[B, C])$, for all $n \times n$-matrices $A, B, C$. Thus, for any finite dimensional Jacobi algebra $A$ which is also a subalgebra of $\mathrm{M}_{n}(k)$ and a Lie subalgebra of $\operatorname{gl}(n, k)$, the restriction of the trace map $\operatorname{Tr}_{\mid A}$ is an integral on $A$.

(4) Let $u \in U(A)$ be an invertible element of a Jacobi algebra $A$. Then the map

$$
\int_{A} \longrightarrow \int_{A_{u}}, \quad v \mapsto v_{u}:=v\left(u^{2} \cdot\right)
$$

is a bijection between the integrals on $A$ and those on the $u$-conformal deformation $A_{u}$. Indeed, we can easily prove that $v_{u}(a):=v\left(u^{2} a\right)$ is an integral on $A_{u}$ for any integral $v$ on $A$ and the inverse of the map $v \mapsto v_{u}$ is given by $\mu \mapsto \mu\left(u^{-2} \cdot\right)-$ we note that $\left(A_{u}\right)_{u^{-1}}=A$. We can also prove that an integral $v$ on $A$ remains an integral on $A_{u}$ if and only if $2[u, x]=2 u\left[1_{A}, x\right]$, for all $x \in A$.

(5) Let $J^{2,4}$ be the Jacobi algebra of Example 2.3. Then $v$ is an integral on $J^{2,4}$ if and only if $v(1)=\alpha$ and $v(x)=0$, for some $\alpha \in k$, i.e. $\int_{J^{2,4}} \cong k$.

(6) Let $J_{11}^{3}$ be the Jacobi algebra from Table 1. Then $v$ is an integral on $J_{11}^{3}$ if and only if $v(1)=\alpha, \quad v(x)=v(y)=0$, for some $\alpha \in k$, i.e. $\int_{J_{11}^{3}} \cong k$. 
Lemma 2.15. Let $A$ be a Jacobi algebra. There exists a bijection between $\int_{A}$ and the space of all (symmetric) associative, invariant bilinear forms on $A$, i.e. bilinear maps $B: A \times A \rightarrow k$ satisfying the following compatibility conditions for any $a, b$, $c \in A$ :

$$
B(a b, c)=B(a, b c), \quad B([a, b], c)=B(a,[b, c])
$$

Proof. Any bilinear form $B$ satisfying (2.12) is symmetric since $A$ is a commutative algebra: $B(a, b)=B\left(1_{A}, a b\right)=B\left(1_{A}, b a\right)=B(b, a)$, for all $a, b \in A$. If $v \in \int_{A}$ is an integral on $A$, then $B_{\nu}(a, b):=v(a b)$ is an associative, invariant, bilinear form on $A$; conversely, if $B$ is such a form then $v_{B}: A \rightarrow k, v_{B}(a):=B\left(a, 1_{A}\right)=$ $B\left(1_{A}, a\right)$ is an integral on $A$ and the correspondence $\left(v \mapsto B_{v}, B \mapsto v_{B}\right)$ is clearly bijective.

The equivalences (1) - (2) - (4) in the theorem below can be seen as the Jacobi versions of the classical characterization of Frobenius algebras ([13]). The equivalence (1) - (3) is the Jacobi counterpart of Sullivan's theorem [44] for Hopf algebras: a Hopf algebra $H$ is co-Frobenius if and only if there exists a non-zero integral on $H$.

Theorem 2.16. Let $A$ be a finite dimensional Jacobi algebra. The following are equivalent:

(1) A is a Frobenius Jacobi algebra;

(2) there exists a nondegenerate associative, invariant, bilinear form on A;

(3) there exists a nondegenerate integral on the Jacobi algebra A;

(4) there exists a pair $\left(v, e=\sum e^{1} \otimes e^{2}\right)$, consisting of an integral $v \in \int_{A}$ on $A$ and an element $e=\sum e^{1} \otimes e^{2} \in A \otimes A$ such that for any $a \in A$ we have:

$$
\sum a e^{1} \otimes e^{2}=\sum e^{1} \otimes e^{2} a, \quad \sum v\left(e^{1}\right) e^{2}=\sum e^{1} v\left(e^{2}\right)=1_{A}
$$

We call such a pair $\left(v, e=\sum e^{1} \otimes e^{2}\right) \in \int_{A} \times(A \otimes A)$ a Jacobi-Frobenius pair and $\omega_{A}:=\sum e^{1} e^{2} \in A$ the Euler-Casimir element of $A$.

Proof. (1) $\Leftrightarrow$ (2) Follows from the one-to-one correspondence between the set of all $k$-linear isomorphisms $f: A \rightarrow A^{*}$ and the set of all nondegenerate bilinear forms $B: A \times A \rightarrow k$ given by the two-sided formula $f(a)(b):=: B(a, b)$, for all $a, b \in A$. Under this bijection, the right $A$-module (resp. right Lie $A$-module) maps $f: A \rightarrow A^{*}$ correspond to those bilinear forms $B: A \times A \rightarrow k$ that satisfy the left (resp. right) hand part of (2.12).

(2) $\Leftrightarrow$ (3) Follows from Lemma 2.15 since under the bijective correspondence ( $v \mapsto B_{v}, B \mapsto v_{B}$ ) nondegenerate forms on $A$ correspond to nondegenerate integrals on $A$. We note that the isomorphism of right Jacobi $A$-modules $f=$ $f_{v}: A \rightarrow A^{*}$ associated to a nondegenerate integral $v$ is defined by $f_{v}(a):=v \triangleleft a$, i.e. $f_{v}(a)(x)=v(a x)$, for all $a, x \in A$. Conversely any nondegenerate integral 
$v=v_{f}$ on $A$ arises from an isomorphism of right Jacobi $A$-modules $f: A \rightarrow A^{*}$ via $v_{f}:=f\left(1_{A}\right)$.

(1) $\Leftrightarrow$ (4) Let $\left\{e_{i}, e_{i}^{*} \mid i=1, \cdots, n\right\}$ be a dual basis of $A$ and $f: A \rightarrow A^{*}$ and isomorphism of Jacobi $A$-bimodules. Then, $v:=f\left(1_{A}\right)$ is a Jacobi integral and by a straightforward computations we can see that $\left(f\left(1_{A}\right), e:=\sum_{i=1}^{n} e_{i} \otimes f^{-1}\left(e_{i}^{*}\right)\right.$ is a Jacobi-Frobenius pair. Conversely, if $\left(\nu, e=\sum e^{1} \otimes e^{2}\right)$ is a Jacobi-Frobenius pair then the map $f=f_{v}: A \rightarrow A^{*}, f(a)(b):=v(a b)$, for all $a, b \in A$ is an isomorphism of Jacobi $A$-bimodules with the inverse $f^{-1}: A^{*} \rightarrow A$ given by $f^{-1}\left(a^{*}\right):=\sum a^{*}\left(e^{1}\right) e^{2}$, for all $a^{*} \in A^{*}$.

Remarks 2.17. (1) Integrals on a unital Poisson algebra $A$ are defined exactly as in Definition 2.13 and a Poisson algebra $A$ is called Frobenius if there exists an isomorphism of Poisson modules $A \cong A^{*}$. The Poisson version of Theorem 2.16 has the same statement and we can rephrase this characterization by saying that a Poisson algebra is Frobenius if and only if it is Frobenius when viewed as a Jacobi algebra.

(2) Let $v \in \int_{A}$ be a nondegenerate integral on a finite dimensional Jacobi algebra $A$. It follows from Theorem 2.16 that $\left(A^{*}, 4\right)$ is free as a right $A$-module having $\{v\}$ as a basis, i.e. $A^{*}=v \triangleleft A$. This is the Jacobi version of a well-know result for Hopf algebras ( [44, Corollary 10.6.6]).

(3) Using (1) $\Leftrightarrow(2)$ of Theorem 2.16 we obtain that the abelian Jacobi algebra $A_{0}$ is Frobenius as a Jacobi algebra if and only if $A$ is Frobenius as an associative algebra.

(4) Let $A$ be a finite dimensional Frobenius Jacobi algebra with the JacobiFrobenius pair $\left(v, e=\sum e^{1} \otimes e^{2}\right)$. In the case of associative Frobenius algebras the element $\omega_{A}:=\sum e^{1} e^{2} \in A$ does not depend on the choice of the Jacobi-Frobenius pair and it is called in [1] the Euler (characteristic) element of $A$ since it is the algebraic counterpart of the Euler class of a connected, oriented, finite dimensional and compact manifold. In the context of finite dimensional Lie algebras equipped with an invariant bilinear form, the same element viewed in the enveloping algebra, is called the Casimir element [9, Proposition 11]. These explain the terminology used in (4) of Theorem 2.16.

Examples 2.18. (1) Let $C_{n}$ be the cyclic group of order $n$ and $k$ a field such that char $(k)=p \mid n-1$. Let $A:=k\left[C_{n}\right]$ be the Jacobi algebra with the bracket $\left[c^{i}, c^{j}\right]:=(j-i) c^{j+i}$, for all $i, j=0, \cdots, n-1$. Then $\int_{k\left[C_{n}\right]}=0$. Indeed, let $v \in \int_{k\left[C_{n}\right]}$. By applying (2.11) in $a=b:=c^{0}=1$ and $c:=c^{i}$, we obtain $v\left(c^{i}\right)=0$, for any $i=0, \cdots, n-1$. Since any group algebra $k[G]$ of a finite group $G$ is Frobenius [27], we obtain that $k\left[C_{n}\right]$ is Frobenius as an associative algebra and using Theorem 2.16 we obtain that it is not Frobenius as a Jacobi algebra.

(2) Let $u \in U(A)$ be an invertible element of a Jacobi algebra $A$. Then, the $u$-conformal deformation $A_{u}$ is a Frobenius Jacobi algebra if and only if $A$ is a Frobenius Jacobi algebra. The result follows from Theorem 2.16 and (4) of Example 2.14 since the bijection there preserves the non-degeneration of integrals. 
(3) The equivalence (1) - (3) of Theorem 2.16 provides a very efficient criterion for deciding when a given Jacobi algebra is Frobenius. For instance, the only 2-dimensional Frobenius Jacobi algebras are $J^{2,1}, J^{2,3}$ and $J_{d}^{2}$ : for each of them the linear map $v$ defined by $v(1):=0$ and $v(x):=1$ is a non-degenerate integral. In the same manner, we can easily prove that among the eleven types of 3-dimensional complex Jacobi algebras given in Example 2.4 there are only three Frobenius Jacobi algebras, namely $J_{2}^{3}, J_{3}^{3}$ and $J_{4}^{3}$. For each of them the linear map $v$ defined by $v(1):=a_{1}, v(x):=a_{2}$ and $v(y):=a_{3}$ is a non-degenerate integral, for all $a_{1}, a_{2}$, $a_{3} \in k$ such that $a_{2} a_{3} \neq 0$ and $a_{1} \neq a_{2}+a_{3}$.

We end the section with the following two questions:

Question 1. Does there exist a finite dimensional Jacobialgebra A which is Frobenius both as an associative algebra and as a algebra Lie, but is not Frobenius as a Jacobi algebra?

Question 2. Let $A$ be a finite dimensional Frobenius Jacobi algebra such that the Euler-Casimir element $\omega_{A}$ is invertible in A. Is every right Jacobi A-module completely reducible (i.e. is A semisimple as a Jacobi algebra)?

\section{Unified products for Jacobi algebras}

In this section we shall answer the extending structures ES-problem for Jacobi algebras: i.e. we shall describe and classify all Jacobi algebras containing a given Jacobi algebra $A$ as a subalgebra of a fixed codimension. We start by explaining what we mean by classification within the ES-problem. Let $A$ be a Jacobi algebra, $E$ a vector space such that $A$ is a subspace of $E$ and let $V$ be a fixed complement of $A$ in $E$, i.e. $V$ is a subspace of $E$ such that $E=A+V$ and $A \cap V=\{0\}$. Let $\mathcal{J}(A, E)$ be the category whose objects are all Jacobi algebra structures $\left(\cdot_{E},[-,-]_{E}\right)$ that can be defined on $E$ such that $A$ becomes a Jacobi subalgebra of $\left(E, \cdot_{E},[-,-]_{E}\right)$. A morphism $\varphi:\left({ }_{E},[-,-]_{E}\right) \rightarrow\left({ }^{\prime}{ }_{E},[-,-]_{E}^{\prime}\right)$ in $\mathcal{J}(A, E)$ is a morphism of Jacobi algebras $\varphi:\left(E,{ }_{E},\{-,-\}_{E}\right) \rightarrow\left(E,{ }_{E}^{\prime},\{-,-\}_{E}^{\prime}\right)$ which stabilizes $A$ and co-stabilizes $V$, i.e. the diagram

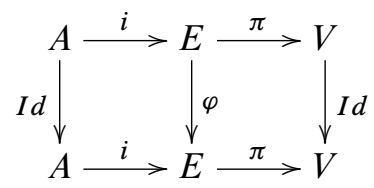

is commutative, where $\pi: E \rightarrow V$ is the canonical projection of $E=A+V$ on $V$ and $i: A \rightarrow E$ is the inclusion map. In this case we say that the Jacobi algebra structures $\left({ }_{E},[-,-]_{E}\right)$ and $\left({ }_{E}^{\prime},[-,-]_{E}^{\prime}\right)$ on $E$ are cohomologous and we denote this by $\left({ }_{E},\{-,-\}_{E}\right) \approx\left({ }_{E}^{\prime},\{-,-\}_{E}^{\prime}\right)$. Any linear map $\varphi$ which makes diagram (3.1) 
commutative is bijective, thus the category $\mathcal{J}(A, E)$ is a groupoid, i.e. any morphism is an isomorphism. In particular, we obtain that $\approx$ is an equivalence relation on the set of objects of $\mathcal{J}(A, E)$ and we denote by $\operatorname{Extd}_{\mathcal{J}}(E, A)$ the set of all equivalence classes, i.e. $\operatorname{Extd}_{\mathcal{J}}(E, A):=\mathcal{J}(A, E) / \approx \operatorname{Extd}_{\mathcal{J}}(E, A)$ is the classifying object for the ES-problem: it classifies all Jacobi algebra structures that can be defined on $E$ containing $A$ as a Jacobi subalgebra up to an isomorphism that stabilizes $A$ and costabilizes $V$. The answer to the ES-problem will be provided by explicitly computing $\operatorname{Extd}_{\mathcal{J}}(E, A)$ for a given Jacobi algebra $A$ and a vector space $E$. From geometrical point of view this means to give the decomposition of the groupoid $\mathcal{J}(A, E)$ into connected components and to indicate a 'point' in each such component. The main result of this section proves that $\operatorname{Extd}_{\mathcal{J}}(E, A)$ is parameterized by a non-abelian cohomological type object $\mathcal{J H}^{2}(V, A)$ that will be explicitly constructed and the bijection between $\mathcal{J H}^{2}(V, A)$ and $\operatorname{Extd}_{\mathcal{J}}(E, A)$ will be indicated.

Definition 3.1. Let $A$ be a Jacobi algebra and $V$ a vector space. An extending datum of $A$ through $V$ is a system $\Upsilon(A, V)=(\triangleleft, \triangleright, f, \cdot,\llcorner,-, \theta,\{-,-\})$ consisting of eight bilinear maps

$$
\begin{array}{rrrr}
\triangleleft: V \times A \rightarrow V, & \triangleright: V \times A \rightarrow A, & f: V \times V \rightarrow A, & \cdot: V \times V \rightarrow V \\
\llcorner: V \times A \rightarrow V, \quad \rightarrow: V \times A \rightarrow A, & \theta: V \times V \rightarrow A, & \{-,-\}: V \times V \rightarrow V
\end{array}
$$

Let $\Upsilon(A, V)=(\triangleleft, \triangleright, f, \cdot, \measuredangle,-, \theta,\{-,-\})$ be an extending datum of a Jacobi algebra $A$ through a vector space $V$. We denote by $A \ltimes \Upsilon(A, V) V=A \ltimes V$ the vector space $A \times V$ together with the multiplication $\bullet$ and the bracket $[-,-]$ defined by:

$$
\begin{array}{r}
(a, x) \bullet(b, y):=(a b+x \triangleright b+y \triangleright a+f(x, y), x \triangleleft b+y \triangleleft a+x \cdot y) \\
{[(a, x),(b, y)]:=([a, b]+x \rightarrow b-y \rightarrow a+\theta(x, y),} \\
x<b-y<a+\{x, y\})
\end{array}
$$

for all $a, b \in A$ and $x, y \in V$. The object $A \ltimes V$ is called the unified product of $A$ and $\Upsilon(A, V)$ if it is a Jacobi algebra with the multiplication defined by (3.2), the unit $\left(1_{A}, 0_{V}\right)$ and the bracket given by (3.3). In this case the extending datum $\Upsilon(A, V)$ is called a Jacobi extending structure of $A$ through $V$.

The next theorem provides the necessary and sufficient conditions that need to be fulfilled by an extending datum $\Upsilon(A, V)$ such that $A \ltimes V$ is a unified product.

Theorem 3.2. Let $A=\left(A, m_{A},[-,-]\right)$ be a Jacobi algebra, $V$ a vector space and $\Upsilon(A, V)=(\triangleleft, \triangleright, f, \cdot, \leftarrow,-, \theta,\{-,-\})$ an extending datum of $A$ through $V$. Then $A \ltimes V$ is a unified product if and only if the following compatibilities hold:

$(J 0)(\triangleleft, \triangleright, f, \cdot)$ is an algebra extending system of the associative algebra A through $V$ and $(\llcorner, \rightarrow, \theta,\{-,-\})$ is a Lie extending system of the Lie algebra $A$ through $V$; 
$(J 1) x \rightarrow(a b)=(x \rightarrow a) b+(x<a) \triangleright b+a(x \rightarrow b)+(x<b) \triangleright a$

$$
-a b\left(x-1_{A}\right)-\left(x<1_{A}\right) \triangleright(a b)
$$

(J2) $x<(a b)=(x<a) \triangleleft b+(x<b) \triangleleft a-\left(x<1_{A}\right) \triangleleft(a b)$

(J3) $x \triangleright[a, b]=[x \triangleright a, b]+(x \triangleleft a) \rightarrow b-a(x \rightarrow b)-(x<b) \triangleright a$ $+(x \triangleright a)\left[1_{A}, b\right]+(x \triangleleft a) \triangleright\left[1_{A}, b\right]$

(J4) $x \triangleleft[a, b]=(x \triangleleft a) \triangleleft b-(x<b) \triangleleft a+(x \triangleleft a) \triangleleft\left[1_{A}, b\right]$

(J5) $\{x, y\} \triangleright a=\theta(x \triangleleft a, y)-a \theta(x, y)+f(y<a, x)$

$-f\left(x \triangleleft a, y<1_{A}\right)-y \rightarrow(x \triangleright a)+x \triangleright(y \rightarrow a)-(x \triangleright a)\left(y \rightarrow 1_{A}\right)$ $-(x \triangleleft a) \triangleright\left(y \rightarrow 1_{A}\right)-\left(y<1_{A}\right) \triangleright(x \triangleright a)$

(J6) $\{x, y\} \triangleleft a=\{x \triangleleft a, y\}-y<(x \triangleright a)+x \triangleleft(y \rightarrow a)+(y<a) \cdot x$

$$
-(x \triangleleft a) \triangleleft\left(y \rightarrow 1_{A}\right)-\left(y<1_{A}\right) \triangleleft(x \triangleright a)-(x \triangleleft a) \cdot\left(y<1_{A}\right)
$$

(J7) $(x \cdot y) \rightarrow a=x \triangleright(y \rightarrow a)+y \triangleright(x \rightarrow a)+f(x<a, y)$

$$
+f(x, y<a)-[f(x, y), a]-f(x, y)\left[1_{A}, a\right]-(x \cdot y) \triangleright\left[1_{A}, a\right]
$$

$(J 8)(x \cdot y)<a=x \cdot(y<a)+(x<a) \cdot y+x \triangleleft(y \rightarrow a)$

$$
+y \triangleleft(x \rightarrow a)-(x \cdot y) \triangleleft\left[1_{A}, a\right]
$$

(J9) $\theta(x \cdot y, z)=x \triangleright \theta(y, z)+y \triangleright \theta(x, z)+z \rightarrow f(x, y)+f(\{x, z\}, y)$

$$
\begin{array}{r}
+f(x,\{y, z\})+f(x, y)\left(z \rightarrow 1_{A}\right)+(x \cdot y) \triangleright\left(z \rightarrow 1_{A}\right) \\
+\left(z<1_{A}\right) \triangleright f(x, y)+f\left(x \cdot y, z<1_{A}\right)
\end{array}
$$

$(J 10)\{x \cdot y, z\}=x \cdot\{y, z\}+\{x, z\} \cdot y+z<f(x, y)+x \triangleleft \theta(y, z)$

$$
\begin{aligned}
+y \triangleleft \theta(x, z)+(x \cdot y) \triangleleft\left(z \rightarrow 1_{A}\right)+ & \left(z<1_{A}\right) \triangleleft f(x, y) \\
+ & (x \cdot y) \cdot\left(z<1_{A}\right)
\end{aligned}
$$

for all $a, b \in A, x, y, z \in V$.

Proof. We have already noticed in Preliminaries that $(A \ltimes V, \bullet)$ is a commutative algebra with unit $\left(1_{A}, 0\right)$ if and only if $(\triangleleft, \triangleright, f, \cdot)$ is an algebra extending system of the algebra $A$ through $V$ and $(A \ltimes V,[-,-])$ is a Lie algebra if and only if $(\llcorner, \rightarrow, \theta,\{-,-\})$ is a Lie extending system of the Lie algebra $A$ through $V$. These are the assumptions from (J0) which from now on we assume to be fulfilled. Then, $(A \ltimes V, \bullet,[-,-])$ is a Jacobi algebra if and only if the following compatibility holds for any $a, b, c \in A$ and $x, y, z \in V$

$$
\begin{aligned}
{[(a, x) \bullet(b, y),(c, z)]=} & (a, x) \bullet[(b, y),(c, z)]+[(a, x),(c, z)] \bullet(b, y) \\
& -(a, x) \bullet(b, y) \bullet\left(\left[1_{A}, c\right]-z \rightarrow 1_{A},-z<1_{A}\right)
\end{aligned}
$$

If we denote the last equation by $(\mathrm{J})$, the proof relies on a detailed analysis of this identity. Since in $A \times V$ we have $(a, x)=(a, 0)+(0, x)$ it follows that (J) holds if and only if it holds for all generators of $A \times V$, i.e. for the set $\{(a, 0) \mid a \in A\} \cup\{(0, x) \mid x \in V\}$. However, since the computations are rather long but straightforward we will only indicate the main steps of the proof, the details 
being available upon request. First, we notice that $(\mathrm{J})$ holds for the triple $(a, 0)$, $(b, 0),(c, 0)$ since $A$ is a Jacobi algebra. The left hand side of (J) evaluated in $(a, 0)$, $(b, 0),(0, x)$ is equal to $(-x \rightarrow(a b),-x<(a b))$ while the right hand side of $(\mathrm{J})$ evaluated in the same triple comes down to:

$$
\begin{aligned}
-((x \rightarrow a) b+ & (x<a) \triangleright b,(x<a) \triangleleft b) \\
- & (a(x \rightarrow b)+(x<b) \triangleright a,(x<b) \triangleleft a) \\
& \quad+\left(a b\left(x \rightarrow 1_{A}\right)+\left(x<1_{A}\right) \triangleright(a b),\left(x<1_{A}\right) \triangleleft(a b)\right)
\end{aligned}
$$

We obtain that (J) holds for the triple $(a, 0),(b, 0),(0, x)$ if and only if (J1) and (J2) hold. Similar computations show the following: (J) holds for the triple $(a, 0),(0, x)$, $(b, 0)$ if and only if (J3) and (J4) hold; (J) holds for the triple $(a, 0),(0, x),(0, y)$ if and only if (J5) and (J6) hold; (J) holds for the triple $(0, x),(0, y),(a, 0)$ if and only if (J7) and (J8) hold and finally, (J) holds for the triple $(0, x),(0, y),(0, z)$ if and only if (J9) and (J10) hold. Moreover, since $A$ is commutative, we observe that the Jacobi compatibility (2.1) holds for the triple $(a, b, c)$ if and only if it holds for the triple $(b, a, c)$. Based on this remark we obtain that $(\mathrm{J})$ holds for the triple $(0, x)$, $(a, 0),(b, 0)$ whenever it holds for $(a, 0),(0, x),(b, 0)$ and $(\mathrm{J})$ holds for the triple $(0, x),(a, 0),(0, y)$ whenever it holds for $(a, 0),(0, x),(0, y)$. The proof is now finished.

From now on a Jacobi extending structure of a Jacobi algebra $A$ through a vector space $V$ will be viewed as a system $\Upsilon(A, V)=(\triangleleft, \triangleright, f, \cdot,\llcorner,-, \theta,\{-,-\})$ satisfying the compatibility conditions (J0)-(J10). We denote by $\mathcal{J E}(A, V)$ the set of all Jacobi algebra extending structures of $A$ through $V$. Theorem 3.2 takes a simplified form at the level of Poisson algebras:

Corollary 3.3. Let $A=\left(A, m_{A},[-,-]\right)$ be a unital Poisson algebra, $V$ a vector space and $\Upsilon(A, V)=(\triangleleft, \triangleright, f, \cdot,-,-\theta,\{-,-\})$ an extending datum of $A$ through $V$. Then $A \ltimes V=(A \ltimes V, \bullet,[-,-])$ is a Poisson algebra with $\left(1_{A}, 0_{V}\right)$ as $a$ unit if and only if the following compatibilities hold for any $a, b \in A, x, y, z \in V$ :

$(P 0)(\triangleleft, \triangleright, f, \cdot)$ is an algebra extending system of the associative algebra $A$ trough $V$ and $(<,-, \theta,\{-,-\})$ is a Lie extending system of the Lie algebra A trough $V$

$(P 1) x \rightarrow(a b)=(x \rightarrow a) b+(x<a) \triangleright b+a(x \rightarrow b)+(x<b) \triangleright a$

(P2) $x<(a b)=(x<a) \triangleleft b+(x<b) \triangleleft a$

(P3) $x \triangleright[a, b]=[x \triangleright a, b]+(x \triangleleft a) \rightarrow b-a(x \rightarrow b)-(x<b) \triangleright a$

(P4) $x \triangleleft[a, b]=(x \triangleleft a)<b-(x<b) \triangleleft a$

(P5) $\{x, y\} \triangleright a=\theta(x \triangleleft a, y)-a \theta(x, y)+f(y<a, x)-y \rightarrow(x \triangleright a)$

$$
+x \triangleright(y \rightarrow a)
$$

(P6) $\{x, y\} \triangleleft a=\{x \triangleleft a, y\}-y<(x \triangleright a)+x \triangleleft(y \rightarrow a)+(y<a) \cdot x$ 
$(P 7)$

$$
\begin{aligned}
(x \cdot y) \rightarrow a=x \triangleright(y-a)+y \triangleright(x \rightarrow a) & +f(x<a, y) \\
& +f(x, y<a)-[f(x, y), a]
\end{aligned}
$$

$(P 8)(x \cdot y)<a=x \cdot(y<a)+(x<a) \cdot y+x \triangleleft(y \rightarrow a)+y \triangleleft(x \rightarrow a)$

(P9) $\theta(x \cdot y, z)=x \triangleright \theta(y, z)+y \triangleright \theta(x, z)+z \rightarrow f(x, y)+f(\{x, z\}, y)$

$$
+f(x,\{y, z\})
$$

(P10) $\{x \cdot y, z\}=x \cdot\{y, z\}+\{x, z\} \cdot y+z<f(x, y)+x \triangleleft \theta(y, z)+y \triangleleft \theta(x, z)$

An extending datum $\Upsilon(A, V)=(\triangleleft, \triangleright, f, \cdot, \leftarrow, \rightarrow, \theta,\{-,-\})$ of a unital Poisson algebra $A$ through a vector space $V$ satisfying the axioms (P0)-(P10) is called a Poisson extending structure of $A$ through $V$ and we denote by $\mathcal{P E}(A, V)$ the set of all Poisson extending structures of $A$ through $V$.

Let $\Upsilon(A, V)=(\triangleleft, \triangleright, f, \cdot, \leftarrow, \rightarrow, \theta,\{-,-\}) \in \mathcal{J E}(A, V)$ be a Jacobi extending structure of a Jacobi algebra $A$ through a vector space $V$. Then $A$ is a Jacobi subalgebra of the unified product $A \ltimes V$ through the identification $A \cong i_{A}(A)=A \times\{0\}$, where $i_{A}: A \rightarrow A \ltimes V, i_{A}(a)=(a, 0)$ is the canonical injection. Conversely, the following result provides the answer to the description part of the ES-problem:

Proposition 3.4. Let $A$ be a Jacobi algebra, $E$ a vector space containing $A$ as a subspace and $\left(*_{E},[-,-]_{E}\right)$ a Jacobi algebra structure on $E$ such that $A$ is a subalgebra of $\left(E, *_{E},[-,-]_{E}\right)$. Then there exists a Jacobi extending structure $\Upsilon(A, V)=(\triangleleft, \triangleright, f, \cdot,\llcorner, \rightarrow, \theta,\{-,-\})$ of $A$ through a subspace $V$ of $E$ and an isomorphism of Jacobi algebras $\left(E, *_{E},[-,-]_{E}\right) \cong A \ltimes V$ that stabilizes $A$ and co-stabilizes $V$.

Proof. Since $k$ is a field, there exists a linear map $p: E \rightarrow A$ such that $p(a)=a$, for all $a \in A$. Then $V:=\operatorname{Ker}(\mathrm{p})$ is a complement of $A$ in $E$. Using the retraction $p$, we define the extending datum $\Upsilon(A, V)=(\triangleleft, \triangleright, f, \cdot,\llcorner, \rightarrow, \theta,\{-,-\})$ of $A$ through $V$ by the following formulas for any $a \in A$ and $x, y \in V$ :

$$
\begin{aligned}
x \triangleright a & :=p\left(x *_{E} a\right), & x \triangleleft a & :=x *_{E} a-p\left(x *_{E} a\right) \\
f(x, y) & :=p\left(x *_{E} y\right), & x \cdot y & :=x *_{E} y-p\left(x *_{E} y\right) \\
x-a & :=p\left([x, a]_{E}\right), & x<a & :=[x, a]_{E}-p\left([x, a]_{E}\right) \\
\theta(x, y) & :=p\left([x, y]_{E}\right), & \{x, y\} & :=[x, y]_{E}-p\left([x, y]_{E}\right)
\end{aligned}
$$

Then by arguments similar to those used for Lie algebras in [5, Theorem 2.4] and associative algebras in [7, Theorem 2.4] we can prove that $\Upsilon(A, V)=(\triangleleft, \triangleright, f, \cdot, \leftarrow$, $\rightarrow, \theta,\{-,-\})$ is a Jacobi extending structure of $A$ through $V$ and the linear map $\varphi: A \ltimes V \rightarrow\left(E, *_{E},[-,-] E\right), \varphi(a, x):=a+x$, is an isomorphism of Jacobi algebras that stabilizes $A$ and co-stabilizes $V$, i.e. the following diagram 
is commutative:

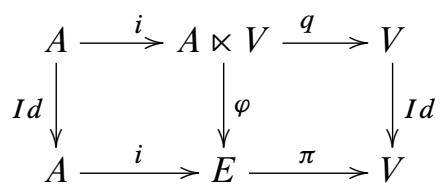

where $q: A \ltimes V \rightarrow V, q(a, x):=x$ is the canonical projection.

Proposition 3.4 reduces the classification of all Jacobi algebra structures on $E$ that contain $A$ as a subalgebra to the classification of all unified products $A \ltimes V$, associated to all Jacobi extending structures $\Upsilon(A, V)=(\triangleleft, \triangleright, f, \cdot,\llcorner, \rightarrow, \theta,\{-,-\})$, for a fixed complement $V$ of $A$ in $E$. First we need the following technical result:

Proposition 3.5. Let $A$ be a Jacobi algebra, $\Upsilon(A, V)=(\triangleleft, \triangleright, f, \cdot, \leftarrow, \rightarrow$, $\theta,\{-,-\})$ and $\Upsilon^{\prime}(A, V)=\left(\triangleleft^{\prime}, \triangleright^{\prime}, f^{\prime}, .^{\prime},\left\llcorner^{\prime},-^{\prime}, \theta^{\prime},\{-,-\}^{\prime}\right)\right.$ two Jacobi extending structures of $A$ through $V$. Let $A \ltimes V$ and $A \ltimes^{\prime} V$ be the unified products associated to the Jacobi extending structures $\Upsilon(A, V)$ and respectively $\Upsilon^{\prime}(A, V)$. The following are equivalent:

(1) There exists $\psi: A \ltimes V \rightarrow A \ltimes^{\prime} V$ a morphism of Jacobi algebras which stabilizes $A$ and co-stabilizes $V$;

(2) $\triangleleft^{\prime}=\triangleleft, \quad \iota^{\prime}=\angle$ and there exists a linear map $r: V \rightarrow A$ such that $\triangleright^{\prime}, f^{\prime}$, $\therefore, \quad \rightarrow^{\prime}, \quad \theta^{\prime}$ and $\{-,-\}^{\prime}$ are implemented by $r$ via the following formulas for any $a \in A, x, y \in V$ :

$$
\begin{array}{rlr}
x \triangleright^{\prime} a & =x \triangleright a+r(x \triangleleft a)-r(x) a, & \\
x \bullet^{\prime} y & =x \cdot y-x \triangleleft r(y)-y \triangleleft r(x) \\
f^{\prime}(x, y) & =f(x, y)+r(x \cdot y)+r(x) r(y)-x \triangleright r(y)-r(x \triangleleft r(y)) \\
x \rightarrow^{\prime} a & =x \rightarrow a+r(x<a)-[r(x), a], & -y \triangleright r(x)-r(y \triangleleft r(x)) \\
\{x, y\}^{\prime} & =\{x, y\}-x<r(y)+y<r(x) \\
\theta^{\prime}(x, y) & =\theta(x, y)+r(\{x, y\})+[r(x), r(y)]+y \rightarrow r(x)-x \rightarrow r(y) \\
& & +r(y<r(x))-r(x<r(y))
\end{array}
$$

Proof. There exists a bijection between the set of all linear maps $\psi: A \times V \rightarrow A \times V$ which stabilize $A$ and co-stabilize $V$ and the set of all linear map $r: V \rightarrow A$ given as follows: $\psi \mapsto r_{\psi}$, where $r_{\psi}(x):=\psi(0, x)$ respectively $r \mapsto \psi_{r}$, where $\psi_{r}(a, x):=(a+r(x), x)$, for all $a \in A$ and $x \in V$. We denote by $\psi_{r}=\psi$, the linear map associated to $r: V \rightarrow A$. We prove that $\psi=\psi_{r}: A \ltimes V \rightarrow A \ltimes^{\prime} V$ is a morphism of Jacobi algebras if and only if the compatibility conditions from (2) hold. Indeed, first we can see that $\psi((a, 0) \bullet(0, x))=\psi(a, 0) \bullet \psi(0, x)$ if and only if $\triangleleft^{\prime}=\triangleleft$ and $x \triangleright^{\prime} a=x \triangleright a+r(x \triangleleft a)$. Taking these two compatibilities into 
account, we can easily see that $\psi((0, x) \bullet(0, y))=\psi(0, x) \bullet \psi(0, y)$ if and only if $x \cdot^{\prime} y=x \cdot y-x \triangleleft r(y)-y \triangleleft r(x)$ and $f^{\prime}(x, y)=f(x, y)+r(x \cdot y)+r(x) r(y)-$ $x \triangleright r(y)-r(x \triangleleft r(y))-y \triangleright r(x)-r(y \triangleleft r(x))$, for all $x, y \in V$. This shows that $\psi=\psi_{r}: A \ltimes V \rightarrow A \ltimes^{\prime} V$ is a morphism of associative algebras if and only if $\triangleleft^{\prime}=\triangleleft$ and the first three compatibility conditions of (2) hold. In a similar fashion, we can prove that $\psi=\psi_{r}: A \ltimes V \rightarrow A \ltimes^{\prime} V$ is a morphism of Lie algebras if and only if $L^{\prime}=\angle$ and the last three compatibility conditions of (2) hold.

The Jacobi algebra morphism $\psi_{r}: A \times V \rightarrow A \times V$ defined in the proof of Proposition 3.5 is bijective. This allows us to introduce the following equivalence relation :

Definition 3.6. Let $A$ be a Jacobi algebra and $V$ a vector space. Two Jacobi extending structures $\Upsilon(A, V)=(\triangleleft, \triangleright, f, \cdot, \leftarrow,-, \theta,\{-,-\})$ and $\Upsilon^{\prime}(A, V)=$ $\left(\triangleleft^{\prime}, \triangleright^{\prime}, f^{\prime}, .^{\prime},\left\llcorner^{\prime},-^{\prime}, \theta^{\prime},\{-,-\}^{\prime}\right)\right.$ are called cohomologous, and we denote this by $\Upsilon(A, V) \approx \Upsilon^{\prime}(A, V)$, if $\triangleleft^{\prime}=\triangleleft, L^{\prime}=\angle$ and there is a linear map $r: V \rightarrow A$ such that $\triangleright^{\prime}, f^{\prime}, \quad{ }^{\prime}, \quad \rightarrow^{\prime}, \quad \theta^{\prime}$ and $\{-,-\}^{\prime}$ are implemented by $r$ via the formulas given in (2) of Proposition 3.5.

The theoretical answer to the ES-problem for Jacobi algebras now follows:

Theorem 3.7. Let $A$ be a Jacobi algebra, $E$ a vector space which contains $A$ as a subspace and $V$ a complement of $A$ in $E$. Then $\approx$ is an equivalence relation on the set $\mathcal{J E}(A, V)$ of all Jacobi algebra extending structures of $A$ through $V$. If we denote by $\mathcal{J H}^{2}(V, A):=\mathcal{J} \mathcal{E}(A, V) / \approx$, then the map $^{1}$

$$
\mathcal{J H}^{2}(V, A) \rightarrow \operatorname{Extd}_{\mathcal{J}}(E, A), \quad \overline{(\triangleleft, \triangleright, f, \cdot,\llcorner,-, \theta,\{-,-\})} \longmapsto A \ltimes V
$$

is bijective, where $A \ltimes V$ is the unified product associated to $A$ and $(\triangleleft, \triangleright, f, \cdot,\llcorner, \rightarrow$, $\theta,\{-,-\})$.

Proof. Proposition 3.5 proves that $\Upsilon(A, V) \approx \Upsilon^{\prime}(A, V)$ if and only if there exists an isomorphism of Jacobi algebras $\psi: A \ltimes V \rightarrow A \ltimes^{\prime} V$ which stabilizes $A$ and costabilizes $V$. This shows that $\approx$ is an equivalence relation on $\mathcal{J E}(A, V)$. The last part follows from this observation together with Theorem 3.2 and Proposition 3.4.

Remark 3.8. The Poisson version of Theorem 3.7 has the following form. Let $A$ be a unital Poisson algebra, $E$ a vector space which contains $A$ as a subspace, $V$ a complement of $A$ in $E$ and let $\mathcal{P E}(A, V)$ be the set of all Poisson extending structures of $A$ through $V$ in the sense of Corollary 3.3. We denote by $\mathcal{P} \mathcal{H}^{2}(V, A):=$ $\mathcal{P E}(A, V) / \approx$, where $\approx$ is the equivalence relation on $\mathcal{P} \mathcal{E}(A, V)$ defined exactly as in Definition 3.6 and by $\operatorname{Extd}_{\mathcal{P}}(E, A)$ the set of all equivalence classes of isomorphism

\footnotetext{
$1 \overline{(\triangleleft, \triangleright, f, \cdot,\llcorner, \longrightarrow, \theta,\{-,-\})}$ denotes the equivalence class of $(\triangleleft, \triangleright, f, \cdot, \longleftarrow, \longrightarrow$, $\theta,\{-,-\})$ via $\approx$.
} 
of all Poisson algebra structures which can be defined on $E$ that contain and stabilize $A$ as a Poisson subalgebra and co-stabilize $V$. Then the map

$$
\mathcal{P} \mathcal{H}^{2}(V, A) \rightarrow \operatorname{Extd}_{\mathcal{P}}(E, A), \quad \overline{\overline{(\triangleleft, \triangleright, f, \cdot,\llcorner,-, \theta,\{-,-\})}} \longmapsto A \ltimes V
$$

is bijective.

Computing the classifying object $\mathcal{J H}^{2}(V, A)$ for a given Jacobi algebra $A$ and a given vector space $V$ is a highly nontrivial problem. However, the first step in computing $\mathcal{J H}^{2}(V, A)$ is suggested by the first part of Definition 3.6: if two Jacobi extending structures $\Upsilon(A, V)=(\triangleleft, \triangleright, f, \cdot,\llcorner, \rightarrow, \theta,\{-,-\})$ and $\Upsilon^{\prime}(A, V)=\left(\triangleleft^{\prime}, \triangleright^{\prime}, f^{\prime}, .^{\prime},\left\llcorner^{\prime}, \rightarrow^{\prime}, \theta^{\prime},\{-,-\}^{\prime}\right)\right.$ are cohomologous, then we must have $\triangleleft^{\prime}=\triangleleft, \iota^{\prime}=\angle$. Thus, in order to compute $\mathcal{J H}^{2}(V, A)$ we can fix the right $A$-module action $\triangleleft$ and the right Lie $A$-module action $\measuredangle$ such that $(V, \triangleleft, \measuredangle)$ is a right Jacobi module as defined in Definition 2.8 - we observe that the compatibility conditions (2.3) and (2.4) of Definition 2.8 coincide with axioms (J2) and (J4) of Theorem 3.2 defining the Jacobi extending structures. Hence, we can decompose the object $\mathcal{J H}^{2}(V, A)$ as follows. Let $A$ be a Jacobi algebra, $V$ a vector space and let $(V, \triangleleft, \angle)$ be a fixed right Jacobi $A$-module. Let $\mathcal{J E}_{(\triangleleft, \sqcup)}(A, V)$ be the set of all $(\triangleleft, \measuredangle)$-Jacobi extending stuctures of $A$ through $(V, \triangleleft, \measuredangle)$ which is the set of all 6-tuples $(\triangleright, f, \cdot, \rightarrow, \theta,\{-,-\})$ consisting of six bilinear maps such that $(\triangleleft, \triangleright, f, \cdot,\llcorner,-, \theta,\{-,-\})$ is a Jacobi extending structure of $A$ through $V$. Two elements $(\triangleright, f, \cdot, \rightarrow, \theta,\{-,-\})$ and $\left(\triangleright^{\prime}, f^{\prime}, .^{\prime}, \rightarrow^{\prime}\right.$, $\left.\theta^{\prime},\{-,-\}^{\prime}\right)$ of $\mathcal{J E}_{(\triangleleft,-)}(A, V)$ are $(\triangleleft, \measuredangle)$-cohomologous and we denote this by $(\triangleright, f, \cdot, \rightarrow, \theta,\{-,-\}) \approx_{l}\left(\triangleright^{\prime}, f^{\prime}, .^{\prime}, \rightarrow^{\prime}, \theta^{\prime},\{-,-\}^{\prime}\right)$ if $(\triangleleft, \triangleright, f, \cdot,\llcorner, \rightarrow$, $\theta,\{-,-\}) \approx\left(\triangleleft, \triangleright^{\prime}, f^{\prime}, .^{\prime}, \measuredangle, \rightarrow^{\prime}, \theta^{\prime},\{-,-\}^{\prime}\right)$. Then $\approx_{l}$ is an equivalence relation on $\mathcal{J E}_{(\triangleleft, \hookrightarrow)}(A, V)$ and we denote by $\mathcal{J H}^{2}((V, \triangleleft, \measuredangle), A)$ the quotient set $\mathcal{J} \mathcal{E}_{(\triangleleft,\llcorner)}(A, V) / \approx_{l}$. Theorem 3.7 and the above considerations provide the following decomposition of $\mathcal{J H}^{2}(V, A)$ :

Corollary 3.9. Let $A$ be a Jacobi algebra and $V$ a vector space. Then

$$
\mathcal{J H}^{2}(V, A)=\sqcup_{(\triangleleft, \hookrightarrow)} \mathcal{J H}^{2}((V, \triangleleft, \sqcup), A)
$$

where the coproduct in the right hand side is in the category of sets over all possible right Jacobi A-module structures $(\triangleleft, \sqcup)$ on $V$.

The decomposition given by (3.4) is a very important step in computing the classifying object $\mathcal{J H}^{2}(V, A)$. However, even computing every object $\mathcal{J H}^{2}((V, \triangleleft, \measuredangle), A)$, for a fixed right Jacobi $A$-module $(V, \triangleleft, \measuredangle)$ is a problem far from being trivial. However the decomposition is important as some of the components in the right hand side of (3.4) might be equal to the empty set as the following example shows. Several explicit examples of computing $\mathcal{J H}^{2}(V, A)$ are provided in Section 4. 
Example 3.10. We consider the trivial right Jacobi $A$-module structure on $V$, that is $x \triangleleft a:=x$ and $x<a:=0$, for all $x \in V, a \in A$. This right Jacobi $A$-module structure on $V$ was denoted by $V_{0}$. If $V \neq\{0\}$, then $\mathcal{J H}^{2}\left(V_{0}, A\right)=\emptyset$. We will prove that in fact the corresponding set $\mathcal{J E}_{(\triangleleft, \leftarrow:=0)}(A, V)$ is empty. Indeed, if $(\triangleright, f, \cdot, \longrightarrow, \theta,\{-,-\}) \in \mathcal{J E}_{(\triangleleft,-:=0)}(A, V)$, then taking into account that $\triangleleft$ acts trivially on $V$, if follows from axiom (A3) that $x \cdot y=x+x \cdot y$, for all $x, y \in V$, thus $V=0$ and we have reached a contraction.

\section{Flag Jacobi algebras. Examples}

In this section we will test the efficiency of Theorem 3.7 and the decomposition given by (3.4) in order to compute $\mathcal{J H}^{2}(V, A)$ for the class of Jacobi algebras defined below:

Definition 4.1. Let $A$ be a Jacobi algebra and $E$ a vector space containing $A$ as a subspace. A Jacobi algebra structure on $E$ is called a flag extending structure of $A$ to $E$ if there exists a finite chain of Jacobi subalgebras of $E$

$$
E_{0}:=A \subset E_{1} \subset \cdots \subset E_{m}:=E
$$

such that $E_{i}$ has codimension 1 in $E_{i+1}$, for all $i=0, \cdots, m-1$. A Jacobi algebra that is a flag extending structure of the Jacobi algebra $k=k_{0}$ is called a flag Jacobi algebra.

All flag extending structures of a given Jacobi algebra $A$ to a vector space $E$ can be completely described by a recursive reasoning where the key step is the case when $A$ has codimension 1 in $E$. This step will provide the description and classification of all unified products $A \ltimes k$ of Jacobi algebras. Then, by replacing $A$ with each of these unified products $A \ltimes k$, we go on with the recursive process in $m$ steps, where $m$ is the codimension of $A$ in $E$. The tool that will play the key role in the description of flag extending structures is the following:

Definition 4.2. Let $A$ be a Jacobi algebra. A Jacobi flag datum of $A$ is a 6-tuple $\left(\Lambda, \Delta, f_{0}, u, \lambda, D\right)$, consisting of four $k$-linear maps $\Lambda, \lambda: A \rightarrow k, \Delta, D: A \rightarrow A$ and two elements $f_{0} \in A$ and $u \in k$ satisfying the following compatibilities for any $a, b \in A$ :

(JF0) $\left(\Lambda, \Delta, f_{0}, u\right)$ is a flag datum of the associative algebra $A$ and $(\lambda, D)$ is a twisted derivation of the Lie algebra $A$;

$$
\begin{aligned}
& \text { (JF1) } D(a b)=D(a) b+a D(b)+\lambda(a) \Delta(b)+\lambda(b) \Delta(a)-\lambda\left(1_{A}\right) \Delta(a b) \\
& -a b D\left(1_{A}\right) \\
& \text { (JF2) } \lambda(a b)=\lambda(a) \Lambda(b)+\lambda(b) \Lambda(a)-\lambda\left(1_{A}\right) \Lambda(a b) \\
& \text { (JF3) } \Delta([a, b])=[\Delta(a), b]+\Lambda(a) D(b)-a D(b)-\lambda(b) \Delta(a)+\Delta(a)\left[1_{A}, b\right] \\
& +\Lambda(a) \Delta\left(\left[1_{A}, b\right]\right)
\end{aligned}
$$


(JF4) $\Lambda([a, b])=\Lambda(a) \Lambda\left(\left[1_{A}, b\right]\right)$

(JF5) $\Delta(D(a))-D(\Delta(a))=\Delta(a) D\left(1_{A}\right)+\Lambda(a) \lambda\left(1_{A}\right) f_{0}-\lambda(a) f_{0}$ $+\Lambda(a) \Delta\left(D\left(1_{A}\right)\right)+\lambda\left(1_{A}\right) \Delta^{2}(a)$

(JF6) $\Lambda(D(a))-\lambda(\Delta(a))=\Lambda(a) \Lambda\left(D\left(1_{A}\right)\right)+\lambda\left(1_{A}\right) \Lambda(a) u-\lambda(a) u$

(JF7) $2 \Delta(D(a))+2 \lambda(a) f_{0}=u D(a)+\left[f_{0}, a\right]+f_{0}\left[1_{A}, a\right]+u \Delta\left(\left[1_{A}, a\right]\right)$

(JF8) $2 \Lambda(D(a))=-\lambda(a) u+u \Lambda\left(\left[1_{A}, a\right]\right)$

(JF9) $D\left(f_{0}\right)+f_{0} D\left(1_{A}\right)+u \Delta\left(D\left(1_{A}\right)\right)+\lambda\left(1_{A}\right) \Delta\left(f_{0}\right)+u \lambda\left(1_{A}\right) f_{0}=0$

(JF10) $\lambda\left(f_{0}\right)+u \Lambda\left(D\left(1_{A}\right)\right)+\lambda\left(1_{A}\right) \Lambda\left(f_{0}\right)+u^{2} \lambda\left(1_{A}\right)=0$

We denote by $\mathcal{J F}(A)$ the set of all Jacobi flag datums of $A$.

For further computations we point out that for any $\left(\Lambda, \Delta, f_{0}, u, \lambda, D\right) \in$ $\mathcal{F} \mathcal{J}(A)$ we have $\Delta\left(1_{A}\right)=0$ and $\Lambda\left(1_{A}\right)=1$.

Proposition 4.3. Let $A$ be a Jacobi algebra. Then there exists a bijection between the set $\mathcal{J E}(A, k)$ of all Jacobi extending structures of $A$ through $k$ and $\mathcal{J} \mathcal{F}(A)$ given such that the unified product $A \ltimes_{\left(\Lambda, \Delta, f_{0}, u, \lambda, D\right)} k$ corresponding to the Jacobi flag datum $\left(\Lambda, \Delta, f_{0}, u, \lambda, D\right) \in \mathcal{J} \mathcal{F}(A)$, denoted by $A_{\left(\Lambda, \Delta, f_{0}, u, \lambda, D\right)}$, is the vector space $A \times k$ with the Jacobi algebra structure given for any $a, b \in A$ and $x, y \in k$ by:

$(a, x) \bullet(b, y)=\left(a b+x \Delta(b)+y \Delta(a)+x y f_{0}, x \Lambda(b)+y \Lambda(a)+x y u\right)$

$[(a, x),(b, y)]=([a, b]+x D(b)-y D(a), x \lambda(b)-y \lambda(a))$

Furthermore, a Jacobi algebra $B$ contains $A$ as a Jacobi subalgebra of codimension 1 if and only if $B \cong A_{\left(\Lambda, \Delta, f_{0}, u, \lambda, D\right)}$, for some Jacobi flag datum $\left(\Lambda, \Delta, f_{0}, u, \lambda, D\right)$ of $A$.

Proof. We have to compute the set of all bilinear maps

$$
\begin{array}{rrr}
\triangleleft: k \times A \rightarrow k, \quad \triangleright: k \times A \rightarrow A, \quad f: k \times k \rightarrow A, & \cdot: k \times k \rightarrow k \\
\llcorner: k \times A \rightarrow k, \quad \rightarrow: k \times A \rightarrow A, \quad \theta: k \times k \rightarrow A, & \{-,-\}: k \times k \rightarrow k
\end{array}
$$

satisfying the compatibility conditions (J0)-(J10) in Theorem 3.2. To start with, the first part of axiom $(J 0)$ tells us that $(\triangleleft, \triangleright, f, \cdot)$ is an algebra extending system of $A$ through $k$ and Example 1.2 proves that there is a bijection between the set of all such maps $(\triangleleft, \triangleright, f, \cdot)$ and the set of all 4-tuples $\left(\Lambda, \Delta, f_{0}, u\right) \in A^{*} \times \operatorname{End}_{k}(A) \times A \times k$ that are flag datums of the associative algebra $A$ : the bijection is given such that the algebra extending system $(\triangleleft, \triangleright, f, \cdot)$ associated to $\left(\Lambda, \Delta, f_{0}, u\right) \in \mathcal{F}(A)$ is defined by the formulas (1.3). Secondly, the last assertion of $(J 0)$ tells us that $(<,-, \theta,\{-,-\})$ is a Lie extending system of the Lie algebra $A$ through the vector space $k$ and Example 1.4 shows that there is a bijection between this set 
and the space $\operatorname{Tw} \operatorname{Der}(A)$ of all twisted derivations of $A$; the bijection is given such that the Lie extending system $(\angle, \rightarrow, \theta,\{-,-\})$ associated to a twisted derivation $(\lambda, D) \in \operatorname{Tw} \operatorname{Der}(A)$ is defined by the formulas (1.5). Hence, there exists a bijection between the set of all bilinear maps $(\triangleleft, \triangleright, f, \cdot, \leftarrow, \rightarrow, \theta,\{-,-\})$ satisfying $(\mathrm{J} 0)$ and the set of all 6-tuples $\left(\Lambda, \Delta, f_{0}, u, \lambda, D\right)$ as defined in Definition 4.2 satisfying the compatibility conditions (JF0) and the bijection is given by the formulas (1.3)-(1.5). The rest of the proof is a long but straightforward computation which shows that, under this bijection, the compatibility conditions (J1)-(J10) of Theorem 3.2 take the equivalent forms given by (JF1)-(JF10). Finally, the Jacobi algebra defined by (4.2)-(4.3) is exactly the associated unified product $A \ltimes k$ defined by (3.2)-(3.3) written in this context. Finally, the last statement follows from the first part and Proposition 3.4.

Let $\left\{e_{i} \mid i \in I\right\}$ be a basis of a Jacobi algebra $A$ and $\left(\Lambda, \Delta, f_{0}, u, \lambda, D\right) \in$ $\mathcal{J F}(A)$ a Jacobi flag datum. Then $A_{\left(\Lambda, \Delta, f_{0}, u, \lambda, D\right)}$ is the Jacobi algebra having $\left\{E, e_{i} \mid i \in I\right\}$ as a basis with the multiplication and the bracket defined for any $i \in I$ by:

$$
\begin{array}{rrr}
e_{i} \bullet e_{j}:=e_{i} \cdot e_{j}, & E \bullet e_{i}=e_{i} \bullet E:=\Delta\left(e_{i}\right)+\Lambda\left(e_{i}\right) E, & E^{2}:=f_{0}+u E \\
{\left[e_{i}, e_{j}\right]:=\left[e_{i}, e_{j}\right]_{A}, \quad} & {\left[E, e_{i}\right]:=D\left(e_{i}\right)+\lambda\left(e_{i}\right) E}
\end{array}
$$

where $\cdot A$ (resp. $[-,-]_{A}$ ) is the multiplication (resp. the bracket) on $A$. The existence of these Jacobi algebras depends on the Jacobi algebra $A$. An interesting fact is the following:

Corollary 4.4. Let $A$ be a perfect Poisson algebra, i.e. $[A, A]=A$. Then, there is no Jacobi algebra which contains $A$ as a subalgebra of codimension 1.

Proof. It follows from Proposition 4.3 if we prove that the set $\mathcal{J F}(A)$ is empty. Indeed, let $\left(\Lambda, \Delta, f_{0}, u, \lambda, D\right) \in \mathcal{J F}(A)$. Since $\left[1_{A}, a\right]=0$, for all $a \in A$, it follows from axiom (JF4) that $\Lambda([a, b])=0$, for all $a, b \in A$. As $A$ is perfect as a Lie algebra, we obtain that $\Lambda(x)=0$, for any $x \in A$, contrary to axiom (FA1) which ensures that $\Lambda\left(1_{A}\right)=1$.

Remark 4.5. A basic invariant of a finite dimensional Lie algebra $\mathfrak{g}$ is the Schur invariant defined by $\alpha(\mathfrak{g}):=$ the maximal dimension of an abelian subalgebra of $\mathfrak{g}$. There is a vast literature devoted to computing this number for several classes of Lie algebras such as (semi)simple, (super)solvable, etc. - see [12,14] and their references. Lie algebras for which $\alpha(\mathfrak{g})=\operatorname{dim}(\mathfrak{g})-1$ are fully described in [19]: below we give the Jacobi algebra version of this result. For a finite dimensional Jacobi algebra $J$ we define the Schur invariant by the formula:

$$
\alpha(J):=\max \{\operatorname{dim}(A) \mid A \text { is an abelian Jacobi subalgebra of } J\}
$$


Using Proposition 4.3 we obtain that a $(n+1)$-dimensional Jacobi algebra $J$ has $\alpha(J)=\operatorname{dim}(J)-1$ if and only if $J \cong A_{\left(\Lambda, \Delta, f_{0}, u, \lambda, D\right)}$, where $A$ is an $n$-dimensional algebra with basis $\left\{e_{i} \mid i=1, \ldots, n\right\}$ and $A_{\left(\Lambda, \Delta, f_{0}, u, \lambda, D\right)}$ is the Jacobi algebra having $\left\{E, e_{i} \mid i=1, \ldots, n\right\}$ as a basis and the multiplication and the bracket is given for any $i, j=1, \ldots n$ by:

$$
\begin{aligned}
e_{i} \bullet e_{j} & :=e_{i} \cdot{ }_{A} e_{j}, & E \bullet e_{i}=e_{i} \bullet E & :=\Delta\left(e_{i}\right)+\Lambda\left(e_{i}\right) E, \\
E^{2} & :=f_{0}+u E, & {\left[E, e_{i}\right] } & :=D\left(e_{i}\right)+\lambda\left(e_{i}\right) E
\end{aligned}
$$

for some 6-tuple $\left(\Lambda, \Delta, f_{0}, u, \lambda, D\right) \in \mathcal{J F}(A)$ with $(D, \lambda) \neq(0,0)$. We mention that in this case the axioms (JF0)-(JF10) which need to be fulfilled by the 6-tuples $\left(\Lambda, \Delta, f_{0}, u, \lambda, D\right)$ take a simplified form as the Lie bracket on $A$ is trivial.

Now we will classify the algebras $A_{\left(\Lambda, \Delta, f_{0}, u, \lambda, D\right)}$ by providing the first explicit classification result of the ES-problem for Jacobi algebras:

Theorem 4.6. Let $A$ be a Jacobi algebra of codimension 1 in the vector space $E$. Then there exist a bijection

$$
\operatorname{Extd}_{\mathcal{J}}(E, A) \cong \mathcal{J H}^{2}(k, A) \cong \mathcal{J} \mathcal{F}(A) / \equiv
$$

where $\equiv$ is the equivalence relation on the set $\mathcal{J} \mathcal{F}(A)$ of all Jacobi flag datums of $A$ defined as follows: $\left(\Lambda, \Delta, f_{0}, u, \lambda, D\right) \equiv\left(\Lambda^{\prime}, \Delta^{\prime}, f_{0}^{\prime}, u^{\prime}, \lambda^{\prime}, D^{\prime}\right)$ if and only if $\Lambda^{\prime}=\Lambda, \lambda^{\prime}=\lambda, u^{\prime}=u$ and there exists $\alpha \in A$ such that for any $a \in A$ we have:

$$
\begin{aligned}
\Delta^{\prime}(a) & =\Delta(a)+\Lambda(a) \alpha-a \alpha \\
f_{0}^{\prime} & =f_{0}+\alpha^{2}+u \alpha-2 \Lambda(\alpha) \alpha-2 \Delta(\alpha) \\
D^{\prime}(a) & =D(a)+\lambda(a) \alpha-[\alpha, a]
\end{aligned}
$$

The bijection between $\mathcal{J} \mathcal{F}(A) / \equiv$ and $\operatorname{Extd}_{\mathcal{J}}(E, A)$ is given by

$$
\overline{\left(\Lambda, \Delta, f_{0}, u, \lambda, D\right)} \mapsto A_{\left(\Lambda, \Delta, f_{0}, u, \lambda, D\right)},
$$

where $\overline{\left(\Lambda, \Delta, f_{0}, u, \lambda, D\right)}$ is the equivalence class of $\left(\Lambda, \Delta, f_{0}, u, \lambda, D\right)$ via the relation $\equiv$.

Proof. Let $\left(\Lambda, \Delta, f_{0}, u, \lambda, D\right),\left(\Lambda^{\prime}, \Delta^{\prime}, f_{0}^{\prime}, u^{\prime}, \lambda^{\prime}, D^{\prime}\right) \in \mathcal{J F}(A)$ and $\Upsilon(A, V)$, respectively $\Upsilon^{\prime}(A, V)$ be the corresponding Jacobi algebra extending structures. Since $\operatorname{dim}_{k}(V)=1$, any linear map $r: V \rightarrow A$ is uniquely determined by an element $\alpha \in A$ such that $r(x)=\alpha$, where $\{x\}$ is a basis in $V$. We can easily see that the compatibility conditions from Proposition 3.5 applied to Jacobi flag datums take precisely the form given in the statement and hence the proof follows from Theorem 3.7 and Proposition 4.3. 
Remark 4.7. In practice, in order to compute the quotient set $\mathcal{J F}(A) / \equiv$ constructed in Theorem 4.6 we shall use the decomposition (3.4) obtained in Corollary 3.9 by going through the following steps. First of all, we shall fix a pair $(\Lambda, \lambda) \in A^{*} \times A^{*}$, consisting of an associative algebra map $\Lambda: A \rightarrow k$ and a Lie algebra map $\lambda: A \rightarrow k$ satisfying the compatibility conditions (2.8). Secondly, for a given such pair $(\Lambda, \lambda)$ we fix a scalar $u \in k$ and compute the set $\mathcal{J F}_{(\Lambda, \lambda)}^{u}(A)$ consisting of all triples $\left(\Delta, f_{0}, D\right) \in \operatorname{End}_{k}(A) \times A \times \operatorname{End}_{k}(A)$ such that $\left(\Lambda, \Delta, f_{0}, u, \lambda, D\right)$ is a Jacobi flag datum of $A$. Two triples $\left(\Delta, f_{0}, D\right)$ and $\left(\Delta^{\prime}, f_{0}^{\prime}, D^{\prime}\right) \in \mathcal{J F}_{(\Lambda, \lambda)}^{u}(A)$ are equivalent and we denote this by $\left(\Delta, f_{0}, D\right) \equiv_{(\Lambda, \lambda)}^{u}\left(\Delta^{\prime}, f_{0}^{\prime}, D^{\prime}\right)$ if and only if there exists $\alpha \in A$ such that (4.7)-(4.9) hold. Finally, we compute the quotient set $\mathcal{J F}_{(\Lambda, \lambda)}^{u}(A) / \equiv_{(\Lambda, \lambda)}^{u}$ $-\mathcal{J H}^{2}(k, A)$ will be the coproduct of these quotients sets over all triples $(\Lambda, \lambda, u)$ - and then we list the isomorphism classes of the associated Jacobi algebras $A_{\left(\Lambda, \Delta, f_{0}, u, \lambda, D\right)}$ using (4.4)-(4.5). To conclude, using Theorem 4.6 and Corollary 3.9, we obtain:

Corollary 4.8. Let A be a Jacobi algebra. Then:

$$
\mathcal{J H}^{2}(k, A) \cong \sqcup_{(\Lambda, \lambda)}\left(\sqcup_{u}\left(\mathcal{J F}_{(\Lambda, \lambda)}^{u}(A) / \equiv_{(\Lambda, \lambda)}^{u}\right)\right)
$$

where the coproducts in the right hand side are made in the category of sets over all possible pairs $(\Lambda, \lambda)$ consisting of an associative algebra map $\Lambda: A \rightarrow k$ and a Lie algebra map $\lambda: A \rightarrow k$ satisfying (2.8) and over all scalars $u \in k$.

Remark 4.9. Let $A$ be a Poisson algebra. The Poisson algebra version of Theorem 4.6 and Corollary 4.8 for computing $\mathcal{P H}^{2}(k, A)$ defined in Remark 3.8 are obtained as follows. First, we define the set $\mathcal{P F}(A)$ of all Poisson flag datums of $A$ : it coincides with the set of all 6-tuples $\left(\Lambda, \Delta, f_{0}, u, \lambda, D\right)$, consisting of four $k$-linear maps $\Lambda$, $\lambda: A \rightarrow k, \Delta, D: A \rightarrow A$ and two elements $f_{0} \in A$ and $u \in k$ satisfying the following compatibilities for any $a, b \in A$ :

(PF0) $\left(\Lambda, \Delta, f_{0}, u\right)$ is a flag datum of the associative algebra $A$ and $(\lambda, D)$ is a twisted derivation of the Lie algebra $A$;

(PF1) $D(a b)=D(a) b+a D(b)+\lambda(a) \Delta(b)+\lambda(b) \Delta(a)$

(PF2) $\lambda(a b)=\lambda(a) \Lambda(b)+\lambda(b) \Lambda(a)$

(PF3) $\Delta([a, b])=[\Delta(a), b]+\Lambda(a) D(b)-a D(b)-\lambda(b) \Delta(a)$

(PF4) $\Lambda([a, b])=0, \quad D\left(f_{0}\right)=0, \quad \lambda\left(f_{0}\right)=0$

(PF5) $D(\Delta(a))-\Delta(D(a))=\lambda(a) f_{0}, \quad \lambda(\Delta(a))-\Lambda(D(a))=\lambda(a) u$

(PF6) $2 \Delta(D(a))+2 \lambda(a) f_{0}=u D(a)+\left[f_{0}, a\right], \quad 2 \Lambda(D(a))=-\lambda(a) u$

Then there exists a bijection $\mathcal{P H}^{2}(k, A) \cong \mathcal{P F}(A) / \equiv$, where $\equiv$ is the equivalence relation defined exactly as in Theorem 4.6, but on the set $\mathcal{P} \mathcal{F}(A)$ of all Poisson flag datums of the Poisson algebra $A$. 


\begin{tabular}{|c|c|c|}
\hline Jacobi algebra & Algebra structure & Lie bracket \\
\hline $\begin{array}{l}J_{\left(\lambda_{1}, \lambda_{2}, u\right)}^{3,1} \\
\left(\lambda_{1}, \lambda_{2}, u\right) \in k \times k^{*} \times k\end{array}$ & $\begin{array}{c}x^{2}=0 \\
y x=x y=2^{-1} u x \\
y^{2}=-4^{-1} u^{2}+u y\end{array}$ & $\begin{array}{l}{[y, 1]=-2^{-1} \lambda_{1} u+\lambda_{1} y} \\
{[y, x]=-2^{-1} \lambda_{2} u+\lambda_{2} y}\end{array}$ \\
\hline $\begin{array}{l}J_{\left(\lambda_{1}, u, f\right)}^{3,2} \\
\left(\lambda_{1}, u, f\right) \in k^{*} \times k^{2}\end{array}$ & $\begin{array}{c}x^{2}=0 \\
y x=x y=2^{-1} u x \\
y^{2}=-4^{-1} u^{2}+f x+u y\end{array}$ & {$[y, 1]=-2^{-1} \lambda_{1} u+\lambda_{1} y$} \\
\hline$J_{\delta}^{3,3}, \delta \in k^{*}$ & $\begin{array}{c}x^{2}=0, y^{2}=\delta^{2} \\
y x=x y=\delta x\end{array}$ & abelian \\
\hline$J_{u}^{3,4}, u \in k^{*}$ & $\begin{array}{c}x^{2}=0, y^{2}=u y \\
y x=x y=0\end{array}$ & abelian \\
\hline $\begin{array}{l}J_{\left(u, f, d_{1}, d_{2}\right)}^{3,} \\
\left(u, f, d_{1}, d_{2}\right) \in k^{4}\end{array}$ & $\begin{array}{c}x^{2}=0 \\
y x=x y=2^{-1} u x \\
y^{2}=-4^{-1} u^{2}+f x+u y\end{array}$ & $\begin{array}{l}{[y, 1]=d_{1} x} \\
{[y, x]=d_{2} x}\end{array}$ \\
\hline
\end{tabular}

Table 2. Flag Jacobi algebras over $J^{2,1}$.

Now, we will illustrate by examples the efficiency of Corollary 4.8 in classifying flag Jacobi algebras. The strategy followed will be that of Remark 4.7 imposed by the decomposition of $\mathcal{J H}^{2}(k, A)$ given in (4.10). Moreover, if $A$ is a Poisson algebra we will also describe $\mathcal{P} \mathcal{H}^{2}(k, A)$ in order to illustrate the difference between it and $\mathcal{J H}^{2}(k, A)$. The model is given below and we make the following convention: all undefined bracket or multiplication of two elements of a basis is zero. Let $J^{2,1}$ be the 2-dimensional Jacobi algebra of Example 2.3.

Proposition 4.10. Let $k$ be a field of characteristic $\neq 2$. Then:

$$
\mathcal{J} \mathcal{H}^{2}\left(k, J^{2,1}\right) \cong\left(k \times k^{*} \times k\right) \sqcup\left(k^{*} \times k^{2}\right) \sqcup k^{*} \sqcup k^{*} \sqcup k^{4}
$$

and the equivalence classes of all 3-dimensional flag Jacobi algebra over $J^{2,1}$ are the Jacobi algebras $J_{\left(\lambda_{1}, \lambda_{2}, u\right)}^{3,1}, J_{\left(\lambda_{1}, u, f\right)}^{3,2}, J_{\delta}^{3,3}, J_{u}^{3,4}, J_{\left(u, f, d_{1}, d_{2}\right)}^{3,5}$ having $\{1, x, y\}$ as a basis and the multiplication and the bracket defined in Table 2 .

Proof. To start with, we should notice that there is only one algebra map $\Lambda: J^{2,1} \rightarrow k$ namely the one given by $\Lambda(1)=1$ and $\Lambda(x)=0$. We denote $\lambda(1)=\lambda_{1}$ and $\lambda(x)=\lambda_{2}$, for some $\left(\lambda_{1}, \lambda_{2}\right) \in k^{2}$. Then $\lambda: J^{2,1} \rightarrow k$ is a Lie algebra map (the Lie brackets on $J^{2,1}$ and $k$ are both abelian) and moreover (2.8) is fulfilled. Thus, the set of all pairs $(\Lambda, \lambda)$ consisting of an algebra map $\Lambda: J^{2,1} \rightarrow k$ and a Lie algebra map $\lambda: J^{2,1} \rightarrow k$ satisfying (2.8) is in bijection with $k^{2}$. From now on we fix a pair $\left(\lambda_{1}, \lambda_{2}\right) \in k^{2}$ and a scalar $u \in k$. We will describe the set $\mathcal{J F}_{(\Lambda, \lambda)}^{u}\left(J^{2,1}\right)$ consisting of all triples $\left(\Delta, f_{0}, D\right)$ such that $\left(\Lambda, \Delta, f_{0}, u, \lambda, D\right)$ is a Jacobi flag datum of $J^{2,1}$. We identify the $k$-linear maps $\Delta$ and $D: J^{2,1} \rightarrow J^{2,1}$ 
with their associated matrices with respect to the basis $\{1, x\}$ :

$$
\Delta=\left(\begin{array}{ll}
\Delta_{11} & \Delta_{12} \\
\Delta_{21} & \Delta_{22}
\end{array}\right) \quad D=\left(\begin{array}{ll}
d_{11} & d_{12} \\
d_{21} & d_{22}
\end{array}\right) \quad f_{0}=f_{1}+f_{2} x
$$

for some $\left(\Delta_{i j}\right) \in k^{4},\left(d_{i j}\right) \in k^{4},\left(f_{1}, f_{2}\right) \in k^{2}$. We have to determine all these scalars such that axioms (JF0)-(JF10) are fulfilled for a fixed triple $\left(\lambda_{1}, \lambda_{2}, u\right) \in k^{3}$. Since this comes down to a laborious computation we will only provide a sketch of it. For instance, we can easily obtain that axiom (JF0) holds for a fixed triple $\left(\lambda_{1}, \lambda_{2}, u\right) \in k^{3}$ if and only if $\left(\Delta, f_{0}, D\right)$ is given by

$$
\Delta=\left(\begin{array}{ll}
0 & 0 \\
0 & \delta
\end{array}\right) \quad D=\left(\begin{array}{ll}
d_{11} & d_{12} \\
d_{21} & d_{22}
\end{array}\right) \quad f_{0}=\delta(\delta-u)+f x
$$

for some $\delta, f \in k$ and $\left(d_{i j}\right) \in k^{4}$ such that $\lambda_{1} d_{12}=\lambda_{2} d_{11}$ and $\lambda_{1} d_{22}=\lambda_{2} d_{21}$. We continue in similar manner by testing the remaining compatibilities: for instance (JF2) and (JF4) hold automatically for such a triple $\left(\Delta, f_{0}, D\right)$ while we can easily see that (JF1) (resp. (JF3)) holds if and only if $d_{12}=-\lambda_{2} \delta$ (resp. $d_{11}=-\lambda_{1} \delta$ ). On the other hand we can prove that (JF5) holds if and only if $\lambda_{2} f=0$ and $\lambda_{2} \delta(2 \delta-u)=0$. Moreover, (JF8) holds if and only if $d_{11}=-2^{-1} \lambda_{1} u$ and $\left.d_{12}=-2^{-1} \lambda_{2} u\right)$. By eliminating the redundant compatibilities we can conclude that axioms (JF0)-(JF10) are fulfilled if and only if the triple $\left(\Delta, f_{0}, D\right)$ given by (4.12) satisfies the following equations:

$$
\begin{gathered}
d_{11}=-\lambda_{1} \delta=-2^{-1} \lambda_{1} u, \quad d_{12}=-\lambda_{2} \delta=-2^{-1} \lambda_{2} u, \quad \lambda_{1} d_{22}=\lambda_{2} d_{21} \\
\lambda_{2} f=\lambda_{2}(2 \delta-u)=\lambda_{1} \delta(2 \delta-u)=f d_{22}=0 \\
d_{21}(2 \delta-u)=d_{22}(2 \delta-u)=0, \quad \lambda_{1}\left(2 \delta^{2}+\delta u-u^{2}\right)=0
\end{gathered}
$$

Now, based on (4.14) we can decompose the set of all Jacobi flag datums of $J^{2,1}$ as follows

$$
\mathcal{J F}\left(J^{2,1}\right)=J_{1} \sqcup J_{2} \sqcup J_{3}
$$

where $J_{1}$ are those flag datums corresponding to $\lambda_{2} \neq 0, J_{2}$ are associated to the case $\lambda_{2}=0$ and $\lambda_{1} \neq 0$ while $J_{3}$ correspond to the case $\lambda_{2}=\lambda_{1}=0$.

In what follows we only provide details for the first case, namely the one corresponding to $\lambda_{2} \neq 0$ - the other two cases are settled using an analogous treatment. If we denote $d_{22}:=d \in k$, then equations (4.13)-(4.15) hold if and only if $f=0, \delta=2^{-1} u$ and $d_{21}=\lambda_{2}^{-1} \lambda_{1} d$. Thus, $J_{1} \cong k \times k^{*} \times k^{2}$ and the bijection is given such that the Jacobi flag datum $\left(\Lambda, \Delta, f_{0}, u, \lambda, D\right) \in \mathcal{J F}\left(J^{2,1}\right)$ associated to $\left(\lambda_{1}, \lambda_{2}, u, d\right) \in k \times k^{*} \times k^{2}$ is given by

$$
\begin{gathered}
\Lambda(1)=1, \quad \Lambda(x)=0, \quad \lambda(1)=\lambda_{1}, \quad \lambda(x)=\lambda_{2} \neq 0 \\
\Delta=\left(\begin{array}{cc}
0 & 0 \\
0 & 2^{-1} u
\end{array}\right) \quad D=\left(\begin{array}{cc}
-2^{-1} \lambda_{1} u & -2^{-1} \lambda_{2} u \\
\lambda_{2}^{-1} \lambda_{1} d & d
\end{array}\right) \quad f_{0}=-4^{-1} u^{2}
\end{gathered}
$$


Now, an elementary computation shows that the equivalence relation of Theorem 4.6 given by (4.7)-(4.9) on the Jacobi flag datums written for the set of triples $k \times k^{*} \times k^{2}$ becomes: $\left(\lambda_{1}, \lambda_{2}, u, d\right) \equiv\left(\lambda_{1}^{\prime}, \lambda_{2}^{\prime}, u^{\prime}, d^{\prime}\right)$ if and only if $\lambda_{1}^{\prime}=\lambda_{1}, \lambda_{2}^{\prime}=\lambda_{2}$, $u^{\prime}=u$ and there exists $q \in k$ such that $d^{\prime}=d+\lambda_{2} q$. Since, $\lambda_{2} \neq 0$, there exists such a $q$, namely $q:=\lambda_{2}^{-1}\left(d^{\prime}-d\right)$. This shows that $\left(\lambda_{1}, \lambda_{2}, u, d\right) \equiv\left(\lambda_{1}, \lambda_{2}, u, 0\right)$, for any $d \in k$ and the quotient set $k \times k^{*} \times k^{2} / \equiv \cong k \times k^{*} \times k \times\{0\} \cong k \times k^{*} \times k$, which is the first component of $\mathcal{J H}^{2}\left(k, J^{2,1}\right)$ in formula (4.11). The 3-parameter Jacobi algebra $J_{\left(\lambda_{1}, \lambda_{2}, u\right)}^{3,1}$ is precisely the unified product $J^{2,1} \ltimes k$ associated to the Jacobi flag datum corresponding to $\left(\lambda_{1}, \lambda_{2}, u, 0\right)$ via the formulas (4.16)-(4.17), with the multiplication and the bracket as defined by (4.4)-(4.5). In a similar fashion we can prove that $J_{2} \cong k^{*} \times k^{3}$ and the bijection is given such that the Jacobi flag datum $\left(\Lambda, \Delta, f_{0}, u, \lambda, D\right) \in \mathcal{J F}\left(J^{2,1}\right)$ associated to $\left(\lambda_{1}, u, f, d\right) \in k^{*} \times k^{3}$ is given by

$$
\begin{gathered}
\Lambda(1)=1, \quad \Lambda(x)=0, \quad \lambda(1)=\lambda_{1} \neq 0, \quad \lambda(x)=0 \\
\Delta=\left(\begin{array}{cc}
0 & 0 \\
0 & 2^{-1} u
\end{array}\right) \quad D=\left(\begin{array}{cc}
-2^{-1} \lambda_{1} u & 0 \\
d & 0
\end{array}\right) \quad f_{0}=-4^{-1} u^{2}+f x
\end{gathered}
$$

and $k^{*} \times k^{3} / \equiv \cong k^{*} \times k^{2}$ since $\left(\lambda_{1}, u, f, d\right) \cong\left(\lambda_{1}^{\prime}, u^{\prime}, f^{\prime}, d^{\prime}\right)$ if and only if $\lambda_{1}^{\prime}=\lambda_{1}, u^{\prime}=u, f^{\prime}=f$ and there exists $q \in k$ such that $d^{\prime}=d+\lambda_{1} q$. The Jacobi algebra $J_{\left(\lambda_{1}, u, f\right)}^{3,2}$ is the unified product $J^{2,1} \ltimes k$ associated to $\left(\lambda_{1}, u, f, d:=0\right)$. Finally, one can show in an analogous manner that $J_{3} \cong\left(k^{*} \sqcup k^{*}\right) \sqcup k^{4}$ and the corresponding Jacobi algebras are the last three families of Table 2: $J_{\delta}^{3,3}, J_{u}^{3,4}$ and respectively $J_{\left(u, f, d_{1}, d_{2}\right)}^{3,5}$.

Remarks 4.11. (1) $J^{2,1}$ is a Poisson algebra and hence we can also compute the classifying object $\mathcal{P} \mathcal{H}^{2}\left(k, J^{2,1}\right)$. By testing which of the Jacobi algebras listed in Proposition 4.10 are Poisson algebras, we obtain that

$$
\mathcal{P} \mathcal{H}^{2}\left(k, J^{2,1}\right) \cong\left(k^{*} \times k\right) \sqcup k^{*} \sqcup k^{*} \sqcup k^{3}
$$

and the equivalence classes of all 3-dimensional flag Poisson algebras over $J^{2,1}$ are the following four families of Poisson algebras $J_{\left(0, \lambda_{2}, u\right)}^{3,1}, J_{\delta}^{3,3}, J_{u}^{3,4}$ and $J_{\left(u, f, 0, d_{2}\right)}^{3,5}$ of Table 2.

(2) Similar to Proposition 4.10 we can describe all flag Jacobi algebras over any 2-dimensional Jacobi algebra listed in Example 2.3. In some cases the computations are straightforward: for instance we can immediately see that $\mathcal{J H}^{2}\left(k, J^{2,2}\right)=\varnothing$ and, if $k \neq k^{2}$, then $\mathcal{J H}^{2}\left(k, J_{d}^{2}\right)=\varnothing$, for all $d \in S \subset k \backslash k^{2}$. Indeed, if $\Lambda: J^{2,2} \rightarrow k$ is an algebra map then, $\Lambda(x)=0$ since $x^{2}=0$ in $J^{2,2}$. By applying axiom (JF4) for $a:=x$ and $b:=1$, we obtain that $\Lambda(1)=0$ and we have reached a contradiction as $\Lambda$ is a unitary algebra map. Thus, we obtained that there is no 3-dimensional Jacobi algebra containing $J^{2,2}$ or $J_{d}^{2}$ as Jacobi subalgebras. The 
remaining two Jacobi algebras of Example 2.3 can be treated in a similar manner and are left to the reader.

The recursive algorithm can be continued in order to describe all 4-dimensional flag Jacobi algebras. For instance, computations similar to those performed in Proposition 4.10 give the following:

Example 4.12. Consider the Jacobi algebra $J_{11}^{3}$ described in Table 1. Then:

$\mathcal{J H}^{2}\left(k, J_{11}^{3}\right) \cong\{*\} \sqcup k \sqcup k^{3} \sqcup k^{2} \sqcup k^{2} \sqcup\left(k^{*} \backslash\{1\}\right) \sqcup k \sqcup k \sqcup k \sqcup k \sqcup\left(k^{*} \backslash\{1\}\right)$

where $\{*\}$ is the singleton set. The equivalence classes of all 4-dimensional flag Jacobi algebras over $J_{11}^{3}$ are the Jacobi algebras with basis $\{1, x, y, z\}$ and the multiplication and the bracket defined below:

$$
\begin{aligned}
& J^{4,1}: x^{2}=x, y^{2}=0, z^{2}=0, x y=y x=0, z y=y z=0, z x=x z=z, \\
& {[y, 1]=y,[z, 1]=2 z,[z, x]=2 \text {; }} \\
& J_{\gamma}^{4,2}: \quad x^{2}=x, y^{2}=0, z^{2}=0, x y=y x=0, z y=y z=0, \\
& z x=x z=\gamma y+z,[y, 1]=y,[z, 1]=z,[z, x]=-\gamma y+z, \\
& \text { where } \gamma \in k \\
& J_{\alpha, u, v}^{4,3}: \quad x^{2}=x, y^{2}=0, x y=y x=0, z x=x z=\alpha-\alpha x+z, \\
& z y=y z=-\alpha y, z^{2}=\alpha^{2}+\alpha u+v x+u z,[y, 1]=y, \\
& {[z, y]=\alpha y, \text { where }(\alpha, u, v) \in k^{3} \text {; }} \\
& J_{\alpha, b}^{4,4}: \quad x^{2}=x, y^{2}=0, x y=y x=0, z x=x z=0, z y=y z=\alpha y, \\
& z^{2}=-\alpha^{2}+\alpha^{2} x+2 \alpha z,[y, 1]=y,[z, y]=b y, \\
& \text { where }(\alpha, b) \in k^{2} \text {; } \\
& J_{\alpha, u}^{4,5}: \quad x^{2}=x, y^{2}=0, x y=y x=0, z x=x z=0, z y=y z=\alpha y, \\
& z^{2}=\alpha(\alpha-u)-\alpha(\alpha-u) x+u z,[y, 1]=y,[z, y]=-\alpha y, \\
& \text { where }(\alpha, u) \in k^{2} \text {; } \\
& J_{\lambda}^{4,6}: x^{2}=x, y^{2}=0, x y=y x=0, z x=x z=x, z y=y z=0, \\
& z^{2}=x,[y, 1]=y,[z, 1]=-\lambda x+\lambda z \\
& \text { where } \lambda \in k^{*}-\{1\} \text {; } \\
& J_{a}^{4,7}: x^{2}=x, y^{2}=0, x y=y x=0, z x=x z=x, z y=y z=0, \\
& z^{2}=x,[y, 1]=y,[z, 1]=-x+a y+z, \text { where } a \in k ; \\
& J_{b}^{4,8}: x^{2}=x, y^{2}=0, x y=y x=0, z x=x z=x, z y=y z=0, \\
& z^{2}=x+b y,[y, 1]=y,[z, 1]=-2^{-1} x+2^{-1} z,
\end{aligned}
$$




$$
\begin{array}{r}
J_{a}^{4,9}: \begin{array}{r}
x^{2}=x, y^{2}=0, x y=y x=0, z x=x z=x, z y=y z=2^{-1} y, \\
z^{2}=-4^{-1}+4^{-1} x+a y+z,[y, 1]=y,[z, y]=-2^{-1} y, \\
{[z, 1]=-4^{-1}-4^{-1} x+2^{-1} z, \text { where } a \in k}
\end{array} \\
J_{a}^{4,10}: \begin{array}{r}
x^{2}=x, y^{2}=0, x y=y x=0, z x=x z=x, z y=y z=2^{-1} y, \\
z^{2}=-4^{-1}+4^{-1} x+z,[y, 1]=y,[z, y]=-2^{-1} y, \\
{[z, 1]=-2^{-1}-2^{-1} x+a y+z, \text { where } a \in k ;}
\end{array} \\
J_{\lambda}^{4,11}: \begin{array}{r}
x^{2}=x, y^{2}=0, x y=y x=0, z x=x z=x, z y=y z=2^{-1} y, \\
z^{2}=-4^{-1}+4^{-1} x+z,[y, 1]=y,[z, y]=-2^{-1} y, \\
{[z, 1]=-2^{-1} \lambda-2^{-1} \lambda x+\lambda z, \text { where } \lambda \in k^{*}-\{1\} .}
\end{array}
\end{array}
$$

\section{Bicrossed products for Poisson algebras. Applications}

In this section we deal with a special case of the unified product for Poisson algebras, namely the bicrossed product and its main applications. Throughout this section the associative algebras are commutative but not necessarily unital. Let $P$ and $Q$ be two given Poisson algebras. A Poisson algebra $R$ factorizes through $P$ and $Q$ if $P, Q$ are Poisson subalgebras of $R$ such that $R=P+Q$ and $P \cap Q=\{0\}$. In this case $Q$ is called a Poisson complement of $P$ in $R$ or a $P$-complement of $R$.

We recall from $[36,37]$ that a matched pair of Lie algebras is a system $(P, Q,\llcorner$, $\rightarrow$ ) consisting of two Lie algebras $P$ and $Q$ and two bilinear maps $\angle: Q \times P \rightarrow Q$ and $\rightarrow: Q \times P \rightarrow P$ such that $(Q, \angle)$ is a right Lie $P$-module, $(P, \rightarrow)$ is a left Lie $Q$-module such that for any $a, b \in P$ and $x, y \in Q$

$$
\begin{aligned}
x \rightarrow[a, b]_{P} & =[x \rightarrow a, b]_{P}+[a, x \rightarrow b]_{P}+(x<a) \rightarrow b-(x<b) \rightarrow a \\
\{x, y\}_{Q}<a & =\{x, y<a\}_{Q}+\{x<a, y\}_{Q}+x<(y \rightarrow a)-y<(x \rightarrow a)
\end{aligned}
$$

The associative algebra counterpart of the matched pair was introduced in [7, Definition 3.6] for non-commutative algebras. A slightly more general definition can be found in [2] where the unitary assumption on the algebras is dropped. In the case of commutative algebras we arrive at the following simplified definition which originates in [8]: a matched pair of commutative algebras is a system $(P, Q, \triangleleft, \triangleright)$ consisting of two commutative algebras $P$ and $Q$ and two bilinear maps $\triangleleft: Q \times P \rightarrow Q$ and $\triangleright: Q \times P \rightarrow P$ such that $(Q, \triangleleft)$ is a right $P$-module, $(P, \triangleright)$ is a left $Q$-module satisfying the following compatibility conditions for any $a, b \in P$ and $x, y \in Q$ :

$$
(x y) \triangleleft a=x \triangleleft(y \triangleright a)+x(y \triangleleft a), \quad x \triangleright(a b)=a(x \triangleright b)+(x \triangleleft b) \triangleright a
$$


It is worth pointing out that these axioms are exactly what remains from (A1)-(A6) of Definition 1.1 if we ask for $\Omega(P, Q)=(\triangleleft, \triangleright, f:=0, \cdot)$ to be an algebra extending system of the algebra $P$ by a vector space $Q$, where $f:=0$ is the trivial map. The concept of a matched pair of Poisson algebras was recently introduced in [41, Theorem 1] - we recall the definition following our notations and terminology since it will be a special case of the axioms (P1)-(P10) appearing in Corollary 3.3.

Definition 5.1. A matched pair of Poisson algebras is a system $(P, Q, \triangleleft, \triangleright,\llcorner, \rightarrow)$ consisting of two Poisson algebras $P$ and $Q$ and four bilinear maps

$$
\triangleleft: Q \times P \rightarrow Q, \quad \triangleright: Q \times P \rightarrow P, \quad\llcorner: Q \times P \rightarrow Q, \quad \rightarrow: Q \times P \rightarrow P
$$

such that $(P, Q, \triangleleft, \triangleright)$ is a matched pair of commutative algebras, $(P, Q, \leftarrow, \rightarrow)$ is a matched pair of Lie algebras satisfying the following compatibility conditions for any $a, b \in P$ and $x, y \in Q$ :

$$
\begin{aligned}
& x \rightarrow(a b)=(x \rightarrow a) b+(x<a) \triangleright b+a(x \rightarrow b)+(x<b) \triangleright a \\
& x<(a b)=(x<a) \triangleleft b+(x<b) \triangleleft a \\
& x \triangleright[a, b]_{P}=[x \triangleright a, b]_{P}+(x \triangleleft a) \rightarrow b-a(x \rightarrow b)-(x<b) \triangleright a \\
& x \triangleleft[a, b]_{P}=(x \triangleleft a)<b-(x<b) \triangleleft a \\
& \{x, y\}_{Q} \triangleright a=x \triangleright(y \rightarrow a)-y \rightarrow(x \triangleright a) \\
& \{x, y\}_{Q} \triangleleft a=\{x \triangleleft a, y\}_{Q}-y<(x \triangleright a)+x \triangleleft(y \rightarrow a)+(y<a) x \\
& (x y) \rightarrow a=x \triangleright(y \rightarrow a)+y \triangleright(x \rightarrow a) \\
& (x y)<a=x(y<a)+(x<a) y+x \triangleleft(y \rightarrow a)+y \triangleleft(x \rightarrow a)
\end{aligned}
$$

The axioms defining a matched pair of Poisson algebras in Definition 5.1 are derived from axioms (P1)-(P10) of Corollary 3.3 if we ask for $\Upsilon(P, Q)=$ $(\triangleleft, \triangleright, f:=0, \cdot,\llcorner, \rightarrow, \theta:=0,\{-,-\})$ to be a Poisson extending structure of the Poisson algebra $P$ through $Q$, where the cocycles $f$ and $\theta$ are both trivial: $f(x, y)=\theta(x, y):=0$, for all $x, y \in Q$.

Let $(P, Q, \triangleleft, \triangleright, \measuredangle,-\rightarrow)$ be a matched pair of Poisson algebras. Then $P \bowtie$ $Q:=P \times Q$ is a Poisson algebra with the multiplication and the bracket defined for any $a, b \in P$ and $x, y \in Q$ by:

$$
\begin{aligned}
& (a, x) \bullet(b, y):=(a b+x \triangleright b+y \triangleright a, x \triangleleft b+y \triangleleft a+x y) \\
& {[(a, x),(b, y)]:=\left([a, b]_{P}+x \rightarrow b-y \rightarrow a, x<b-y<a+\{x, y\}_{Q}\right)}
\end{aligned}
$$

called the bicrossed product associated to the matched pair $(P, Q, \triangleleft, \triangleright, \angle, \rightarrow)$. The bicrossed product of Poisson algebras is exactly the unified product $P \ltimes Q$ associated to a Poisson extending structure of the Poisson algebra $P$ through $Q$ having both cocyles trivial $f=\theta:=0$. The bicrossed product is the construction 
responsible for the so-called factorization problem which formulated at the level of Poisson algebras comes down to the following: for two given Poisson algebras $P$ and $Q$ describe and classify all Poisson algebras which factorize through $P$ and $Q$. The next result is [41, Theorem 1]. For the reader's convenience we also include a short and different proof of it based on Proposition 3.4.

Proposition 5.2. Let $P$ and $Q$ be two given Poisson algebras. A Poisson algebra $R$ factorizes trough $P$ and $Q$ if and only if there exists $(P, Q, \triangleleft, \triangleright,\llcorner,-\rightarrow)$ a matched pair of Poisson algebras such that $R \cong P \bowtie Q$, an isomorphism of Poisson algebras.

Proof. First we observe that any bicrossed product $P \bowtie Q$ factorizes through $P \cong P \times\{0\}$ and $Q \cong\{0\} \times Q$ and, via this identification, $P$ and $Q$ are Poisson subalgebras of $P \bowtie Q$ such that $P \bowtie Q=P+Q$ and $P \cap Q=\{0\}$. Conversely, assume that $R=\left(R, \cdot_{R},[-,-]_{R}\right)$ is a Poisson algebra that factorizes through $P$ and $Q$. Let $\iota: P \rightarrow R$ be the inclusion map and $\pi: R \rightarrow P$ the canonical $k$-linear retraction of $\iota$, i.e. $\pi(p+q):=p$. Since $Q=\operatorname{Ker}(\pi)$ is a Poisson subalgebra of $R$ we obtain that the cocycles $f$ and $\theta: Q \times Q \rightarrow P$ constructed in the proof of Proposition 3.4 are both trivial: i.e. $f(x, y):=\pi(x \cdot R y)=0$ and $\theta(x, y):=\pi\left([x, y]_{R}\right)=0$, for all $x, y \in Q$. Thus, the actions $(\triangleleft, \triangleright,\llcorner, \rightarrow)$ defined for any $a \in P$ and $x \in Q$ by:

$$
\begin{aligned}
x \triangleright a & :=\pi\left(x \cdot_{R} a\right), \quad x \triangleleft a & :=x \cdot_{R} a-\pi\left(x \cdot \cdot_{R} a\right) \\
x \rightarrow a & :=\pi\left([x, a]_{R}\right), \quad x<a & :=[x, a]_{R}-\pi\left([x, a]_{R}\right)
\end{aligned}
$$

make $(P, Q, \triangleleft, \triangleright, \measuredangle, \rightarrow)$ a matched pair of Poisson algebras while $\varphi: P \bowtie$ $Q \rightarrow R, \varphi(a, x):=a+x$ becomes an isomorphism of Poisson algebras.

As we have seen in the proof of Proposition 5.2, if a Poisson algebra $R$ factorizes through $P$ and $Q$ then we can construct a matched pair of Poisson algebras as follows:

$$
x \triangleright a+x \triangleleft a=x a, \quad x \rightarrow a+x<a=[x, a]
$$

for all $a \in P$ and $x \in Q$. Throughout, the above matched pair will be called the canonical matched pair associated with the factorization of $R$ through $P$ and $Q$. Proposition 5.2 allows for a computational reformulation of the factorization problem as follows: for two given Poisson algebras $P$ and $Q$ describe and classify all bicrossed products $P \bowtie Q$ associated to all possible matched pairs $(P, Q, \triangleleft, \triangleright, \measuredangle,-\rightarrow)$. The problem is far from being a trivial one.

Example 5.3. Let $k_{0}$ be the trivial 1-dimensional Poisson algebra with basis $\{X\}$ and $\mathcal{H}$ the 3-dimensional Heisenberg Lie algebra with basis $\left\{H_{1}, H_{2}, H_{3}\right\}$ and the bracket defined by $\left[H_{1}, H_{2}\right]=H_{3}$. $\mathcal{H}$ admits a Poisson algebra structure with the associative multiplication given by $H_{1}^{2}=H_{3}$. It can be easily seen by a straightforward computation that the bicrossed products corresponding to all matched 
pairs of Poisson algebras $\left(k_{0}, \mathcal{H}, \triangleleft, \triangleright, \measuredangle, \rightarrow\right)$ are the 4-dimensional Poisson algebras with basis $\left\{X, H_{1}, H_{2}, H_{3}\right\}$ listed below:

$$
\begin{aligned}
& \mathcal{H}_{(\beta, \alpha, \mu, \eta)}^{1}: \quad X^{2}=0, H_{1}^{2}=H_{3}, X H_{1}=H_{1} X=\alpha H_{3}, X H_{2}=H_{2} X=\beta H_{3}, \\
& {\left[H_{1}, H_{2}\right]=H_{3},\left[H_{1}, X\right]=\mu H_{3},\left[H_{2}, X\right]=\eta H_{3} \text {, }} \\
& \text { for }(\beta, \alpha, \mu, \eta) \in k^{*} \times k^{3} \\
& \mathcal{H}_{(\xi, \mu, \eta)}^{2}: \quad X^{2}=0, H_{1}^{2}=H_{3},\left[H_{1}, H_{2}\right]=H_{3}, \\
& {\left[H_{1}, X\right]=\xi X+\mu H_{2}+\eta H_{3} \text { for }(\xi, \mu, \eta) \in k^{3}} \\
& \mathcal{H}_{(\eta, \mu, \tau)}^{3}: \quad X^{2}=0, H_{1}^{2}=H_{3},\left[H_{1}, H_{2}\right]=H_{3},\left[H_{2}, X\right]=\eta H_{3} \text {, } \\
& {\left[H_{1}, X\right]=\mu H_{2}+\tau H_{3}, \text { for }(\eta, \mu, \tau) \in k^{*} \times k^{2}} \\
& \mathcal{H}_{(\alpha, \mu, \tau, \eta)}^{4}: \quad X^{2}=0, H_{1}^{2}=H_{3}, X H_{1}=H_{1} X=\alpha H_{3},\left[H_{1}, H_{2}\right]=H_{3} \text {, } \\
& {\left[H_{1}, X\right]=\mu H_{2}+\tau H_{3},\left[H_{2}, X\right]=\eta H_{3} \text {, }} \\
& \text { for }(\alpha, \mu, \tau, \eta) \in k^{*} \times k^{3} \\
& \mathcal{H}_{(\xi, \mu, \eta)}^{5}: \quad X^{2}=0, H_{1}^{2}=H_{3},\left[H_{1}, H_{2}\right]=H_{3},\left[H_{1}, X\right]=\mu H_{3}, \\
& {\left[H_{2}, X\right]=\xi X+\eta H_{3}, \text { for }(\xi, \mu, \eta) \in k^{*} \times k^{2}} \\
& \mathcal{H}_{(\xi, \gamma, \mu, \eta)}^{6}: \quad X^{2}=0, H_{1}^{2}=H_{3},\left[H_{1}, H_{2}\right]=H_{3} \text {, } \\
& {\left[H_{1}, X\right]=\xi X+\xi \gamma^{-1} \eta H_{2}+\mu H_{3},} \\
& {\left[H_{2}, X\right]=\gamma X+\eta H_{2}+\xi^{-1}(\xi+1) \eta H_{3},} \\
& \text { for }(\xi, \gamma, \mu, \eta) \in\left(k^{*}\right)^{2} \times k^{2}
\end{aligned}
$$

The rest of the section we deal with the converse of the factorization problem, called the bicrossed descent (or the classification of complements) problem and whose statement was given in the introduction. First we need to introduce the following concept:

Definition 5.4. Let $(P, Q, \triangleleft, \triangleright, \measuredangle, \rightarrow)$ be a matched pair of Poisson algebras. A $k$-linear map $r: Q \rightarrow P$ is called a deformation map of the above matched pair if the following compatibilities hold for all $p, q \in Q$ :

$$
\begin{aligned}
r(p) r(q)-r(p q) & =r(q \triangleleft r(p)+p \triangleleft r(q))-q \triangleright r(p)-p \triangleright r(q) \\
r([p, q])-[r(p), r(q)] & =r(q<r(p)-p<r(q))+p \rightarrow r(q)-q \rightarrow r(p)
\end{aligned}
$$

We denote by $\mathcal{D} \mathcal{M}(P, Q \mid(\triangleleft, \triangleright,\llcorner, \rightarrow))$ the set of all deformation maps of the matched pair $(P, Q, \triangleleft, \triangleright,\llcorner, \rightarrow)$. 
Example 5.5. Consider the following matched pair between $k_{0}$ and $\mathcal{H}$ :

$$
H_{1} \triangleleft X=H_{3}, \quad H_{2} \triangleleft X=H_{3}, \quad H_{1} \triangleleft X=H_{3}, \quad H_{2} \triangleleft X=H_{3}
$$

where the undefined actions are all equal to 0 . The corresponding bicrossed product is the Poisson algebra $\mathcal{H}_{(1,1,1,1)}^{1}$ from Example 5.3. Any deformation map associated to the above matched pair is given as follows:

$$
\begin{gathered}
r_{\left(a_{1}, a_{2}\right)}: \mathcal{H} \rightarrow k_{0}, \quad r_{\left(a_{1}, a_{2}\right)}\left(h_{1}\right)=a_{1} X, \\
r_{\left(a_{1}, a_{2}\right)}\left(h_{2}\right)=a_{2} X, \quad r_{\left(a_{1}, a_{2}\right)}\left(h_{3}\right)=0
\end{gathered}
$$

for some $a_{1}, a_{2} \in k$.

The next result shows that to any deformation map $r$ we can associate a new Poisson algebra called the $r$-deformation and, moreover, all complements of a given Poisson algebra extension $P \subset R$ can be described as $r$-deformations of a given complement.

Theorem 5.6. Let $P$ be a Poisson subalgebra of $R$ and $Q$ a given $P$-complement of $R$ with the associated canonical matched pair $(P, Q, \triangleleft, \triangleright,\llcorner, \rightarrow)$.

(1) Let $r: Q \rightarrow P$ be a deformation map of the above matched pair. Then $Q_{r}:=Q$, as a vector space, with the new Poisson algebra structure defined for any $q, t \in Q$ by:

$$
\begin{aligned}
q \cdot r t & =q t+t \triangleleft r(q)+q \triangleleft r(t) \\
{[q, t]_{r} } & =[q, t]+q<r(t)-t<r(q)
\end{aligned}
$$

is a Poisson algebra called the $r$-deformation of $Q$ and $Q_{r}$ is a $P$-complement of $R$.

(2) $\bar{Q}$ is a P-complement of $R$ if and only if there exists an isomorphism of Poisson algebras $\bar{Q} \cong Q_{r}$, for some deformation map $r: Q \rightarrow P$ of the above canonical matched pair.

Proof. Let $P \bowtie Q$ be the bicrossed product associated to the canonical matched pair $(P, Q, \triangleleft, \triangleright, \angle,-)$. It follows form Proposition 5.2 that $R \cong P \bowtie Q$ as Poisson algebras.

(1) Although this claim can be proven by a very long but straightforward computation, we will provide a different and more natural approach. Given a deformation map $r: Q \rightarrow P$, we consider $f_{r}: Q \rightarrow P \bowtie Q$ to be the $k$-linear map defined for all $q \in Q$ by:

$$
f_{r}(q)=(r(q), q)
$$

It turns out that $\widetilde{Q}:=\operatorname{Im}\left(f_{r}\right)$ is a $P$ complement of $R \cong P \bowtie Q$. 
We start by proving that $\widetilde{Q}$ is a Poisson subalgebra of $P \bowtie Q$. Indeed, for all $p$, $q \in Q$ we have:

$$
\begin{gathered}
(r(p), p)(r(q), q)=\frac{(r(p) r(q)+p \triangleright r(q)+q \triangleright r(p),}{p \triangleleft r(q)+q \triangleleft r(p)+p q)} \\
\stackrel{\stackrel{5.16)}{=}(r(p q+p \triangleleft r(q)+q \triangleleft r(p)),}{p \triangleleft r(q)+q \triangleleft r(p)+p q)} \\
{[(r(p), p),(r(q), q)]=\left(\frac{[r(p), r(q)]+p \rightarrow r(q)-q \rightarrow r(p),}{p<r(q)-q<r(p)+[p, q])}\right.} \\
\stackrel{\stackrel{5.17)}{=}(r([p, q]+p<r(q)-q<r(p)),}{p<r(q)-q<r(p)+[p, q])}
\end{gathered}
$$

Therefore $\widetilde{Q}$ is a Poisson subalgebra of $P \bowtie Q$. Consider now $(p, q) \in P \cap \widetilde{Q}$. Since in particular we have $(p, q) \in \widetilde{Q}$ then $p=r(q)$. As we also have $(r(q), q) \in P$ we obtain $q=0$ and thus $P \cap \widetilde{Q}=\{0\}$. Furthermore, for any $(p, q) \in R=P \bowtie Q$ we can write $(p, q)=(p-r(q), 0)+(r(q), q) \in P+\widetilde{Q}$. Hence, we have proved that $\widetilde{Q}$ is a $P$-complement of $P \bowtie Q$. We are left to prove that $Q_{r}$ and $\widetilde{Q}$ are isomorphic as Poisson algebras. To this end, we denote by $\widetilde{f}_{r}$ the linear isomorphism from $Q$ to $\widetilde{Q}$ induced by $f_{r}$. As we will see, $\widetilde{f}_{r}$ is a Poisson algebra map if we consider $Q$ endowed with the Poisson structures given by (5.16) and (5.17). Indeed, for all $q, t \in Q$ we have:

$$
\begin{aligned}
\bar{f}_{r}(q \cdot r t) & \stackrel{(5.16)}{=} \bar{f}_{r}(q t+t \triangleleft r(q)+q \triangleleft r(t)) \\
& =(r(q t+t \triangleleft r(q)+q \triangleleft r(t)), q t+t \triangleleft r(q)+q \triangleleft r(t)) \\
& \stackrel{(5.14)}{=}(r(q) r(t)+q \triangleright r(t)+t \triangleright r(q), q t+t \triangleleft r(q)+q \triangleleft r(t)) \\
& \stackrel{(5.11)}{=}(r(q), q)(r(t), t)=\bar{f}_{r}(q) \bar{f}_{r}(t)
\end{aligned}
$$

and

$$
\begin{aligned}
\bar{f}_{r}\left([q, t]_{r}\right) & \stackrel{(5.16)}{=} \bar{f}_{r}([q, t]+q<r(t)-t<r(q)) \\
& =(r([q, t]+q<r(t)-t<r(q)),[q, t]+q<r(t)-t<r(q)) \\
& \stackrel{(5.15)}{=}([r(q), r(t)]+q \rightarrow r(t)-t \rightarrow r(q), \\
& \stackrel{(5.12)}{=}[(r(q), q),(r(t), t)]=\left[\bar{f}_{r}(q), \bar{f}_{r}(t)\right]
\end{aligned}
$$

Hence we can conclude that $Q_{r}$ is a Poisson algebra and this finishes the proof. 
(2) Let $\bar{Q}$ be an arbitrary $P$-complement of $R$. As $R=P \oplus Q=P \oplus \bar{Q}$ we can find four $k$-linear maps:

$$
a: Q \rightarrow P, \quad b: Q \rightarrow \bar{Q}, \quad c: \bar{Q} \rightarrow P, \quad d: \bar{Q} \rightarrow Q
$$

such that for all $q \in Q$ and $t \in \bar{Q}$ we have:

$$
q=a(q) \oplus b(q), \quad t=c(t) \oplus d(t)
$$

It follows by an easy computation that $b: Q \rightarrow \bar{Q}$ is an isomorphism of vector spaces. We will denote by $\widetilde{b}: Q \rightarrow P \bowtie Q$ the composition $\widetilde{b}:=i \circ b$, where $\underset{i}{i}: \bar{Q} \rightarrow R=P \bowtie Q$ is the canonical inclusion. Thus, by (5.18) we have $\widetilde{b}(t)=(-a(t), t)$ for all $t \in Q$. We will prove that $r:=-a$ is a deformation map and $\bar{Q} \cong Q_{r}$. Indeed, $\bar{Q}=\operatorname{Im}(b)=\operatorname{Im}(\vec{b})$ is a Poisson subalgebra of $R=P \bowtie Q$ and we have:

$$
\begin{gathered}
(r(q), q)(r(t), t) \stackrel{(5.11)}{=}(r(q) r(t)+q \triangleright r(t)+t \triangleright r(q), \\
q \triangleleft r(t)+t \triangleleft r(q)+q t)=\left(r\left(t^{\prime}\right), t^{\prime}\right) \\
{[(r(q), q),(r(t), t)] \stackrel{(5.12)}{=}([r(q), r(t)]+q \rightarrow r(t)-t \rightarrow r(q),} \\
q<r(t)-t<r(q)+[q, t])=\left(r\left(t^{\prime \prime}\right), t^{\prime \prime}\right)
\end{gathered}
$$

for some $t^{\prime}, t^{\prime \prime} \in Q$. Hence, we have:

$$
r\left(t^{\prime}\right)=r(q) r(t)+q \triangleright r(t)+t \triangleright r(q), \quad t^{\prime}=q \triangleleft r(t)+t \triangleleft r(q)+q t
$$

$r\left(t^{\prime \prime}\right)=[r(q), r(t)]+q \rightarrow r(t)-t \rightarrow r(q), \quad t^{\prime \prime}=q<r(t)-t<r(q)+[q, t]$

By applying $r$ to the second part of (5.19), respectively (5.20), we obtain that $r$ is a deformation map. Moreover, by a straightforward computation using (5.16), (5.17), (5.19) and respectively (5.20) it follows that $b: Q_{r} \rightarrow \bar{Q}$ is a Poisson algebra map and the proof is now finished.

Examples 5.7. Let $k$ be an algebraically closed field of characteristic zero and let $\mathcal{H}_{1,1,1,1}^{1}$ be the bicrossed product described in Example 5.3. For any $\left(a_{1}, a_{2}\right) \in k^{2}$ consider $r_{\left(a_{1}, a_{2}\right)}: \mathcal{H} \rightarrow k_{0}$ the associated deformation map described in Example 5.5. Then, the corresponding $r_{\left(a_{1}, a_{2}\right)}$-deformation $\mathcal{H}_{r_{\left(a_{1}, a_{2}\right)}}$ of the Heisenberg Poisson algebra has the associative algebra structure and the Lie bracket given as follows:

$$
\begin{aligned}
\mathcal{H}_{\left.r_{\left(a_{1}, a_{2}\right.}\right)}: \quad H_{1}^{2}=\left(2 a_{1}+1\right) H_{3}, H_{1} H_{2}= & \left(a_{1}+a_{2}\right) H_{3}, \\
& {\left[H_{1}, H_{2}\right]=\left(a_{2}-a_{1}+1\right) H_{3} }
\end{aligned}
$$


If $a_{1}=a_{2}=0$ then $\mathcal{H}_{\left.r_{(0,0}\right)}$ coincides with $\mathcal{H}$. Moreover, for any $a \in k-\left\{2^{-1}\right\}$ the $r_{(a,-a)}$-deformation $\mathcal{H}_{r_{(a,-a)}}$ is isomorphic to the Heisenberg Poisson algebra $\mathcal{H}$, the isomorphism of Poisson algebras being given by:

$$
\begin{aligned}
& \varphi: \mathcal{H}_{r_{(a,-a)}} \rightarrow \mathcal{H}, \quad \varphi\left(H_{1}\right)=\alpha H_{1}, \\
& \varphi\left(H_{2}\right)=(-2 a+1) \alpha^{-1} H_{2}, \quad \varphi\left(H_{3}\right)=H_{3}
\end{aligned}
$$

where $\alpha$ is a square root of $(2 a+1)$. However, if we consider $a_{1}=1$ and $a_{2}=0$ then we obtain the Poisson algebra $\mathcal{H}_{r_{(1,0)}}$ with the trivial Lie bracket and the multiplication given by: $H_{1}^{2}=3 H_{3}, H_{1} H_{2}=H_{3}$. Therefore, $\mathcal{H}$ and $\mathcal{H}_{r_{(1,0)}}$ are not isomorphic as Poisson algebras as a consequence of not being isomorphic as Lie algebras.

As the previous example shows it, different deformation maps can give rise to isomorphic deformations. Therefore, in order to provide the classification of complements we need to introduce the following:

Definition 5.8. Let $(P, Q, \triangleleft, \triangleright, \measuredangle, \rightarrow)$ be a matched pair of Poisson algebras. Two deformation maps $r, r^{\prime}: Q \rightarrow P$ are called equivalent and we will denote this by $r \sim r^{\prime}$ if there exists $\sigma: Q \rightarrow Q$ a linear automorphism of $Q$ such that for any $q, t \in Q$ we have:

$$
\begin{gathered}
\sigma(q t)-\sigma(q) \sigma(t)=\sigma(q) \triangleleft r^{\prime}(\sigma(t))+\sigma(t) \triangleleft r^{\prime}(\sigma(q)) \\
-\sigma(q \triangleleft r(t))-\sigma(t \triangleleft r(q)) \\
\sigma([q, t])-[\sigma(q), \sigma(t)]=\sigma(q)\left\llcorner r^{\prime}(\sigma(t))-\sigma(t)<r^{\prime}(\sigma(q))\right. \\
+\sigma(t<r(q))-\sigma(q\llcorner r(t))
\end{gathered}
$$

The main result of this section which provides the answer to the bicrossed descent problem now follows:

Theorem 5.9. Let $P$ be a Poisson subalgebra of $R, Q$ a P-complement of $R$ and $(P, Q, \triangleleft, \triangleright, \leftarrow,-\rightarrow)$ the associated canonical matched pair. Then $\sim i s$ an equivalence relation on the $\operatorname{set} \mathcal{D} \mathcal{M}(P, Q \mid(\triangleleft, \triangleright,\llcorner, \rightarrow))$ and the map

$$
\begin{array}{r}
\mathcal{H} \mathcal{A}^{2}(P, Q \mid(\triangleleft, \triangleright, \measuredangle, \rightarrow)):=\mathcal{D} \mathcal{M}(P, Q \mid(\triangleleft, \triangleright,\llcorner, \rightarrow)) / \sim \rightarrow \\
\mathcal{F}(P, R), \\
\bar{r} \mapsto Q_{r}
\end{array}
$$

is a bijection, where $\mathcal{F}(P, R)$ is the set of isomorphism classes of all $P$-complements of $R$. In particular, the factorization index of $P$ in $R$ is computed by the formula:

$$
[R: P]^{f}=\left|\mathcal{H} \mathcal{A}^{2}(P, Q \mid(\triangleleft, \triangleright, \measuredangle, \rightarrow))\right|
$$

Proof. Two deformation maps $r$ and $r^{\prime}$ are equivalent in the sense of Definition 5.8 if and only if the corresponding Poisson algebras $Q_{r}$ and $Q_{r^{\prime}}$ are isomorphic. The conclusion follows by Theorem 5.6. 
In what follows, for any $a \in k$, we denote by $\mathcal{H}_{a}$ the deformation of the Heisenberg Poisson algebra described in Example 5.7 associated to the pair $(0, a) \in k^{2}$. More precisely, the Poisson algebra structure on $\mathcal{H}_{a}$ is as follows:

$$
\mathcal{H}_{a}: \quad H_{1}^{2}=H_{3}, H_{1} H_{2}=a H_{3},\left[H_{1}, H_{2}\right]=(a+1) H_{3}
$$

Our next result provides a classification result for the $k_{0}$-complements of $\mathcal{H}_{1,1,1,1}^{1}$ from Example 5.3. In particular, the deformations $\mathcal{H}_{a}$ provide an infinite family of non-isomorphic three dimensional Poisson algebras - for a similar result in the setting of Lie algebras see [6].

Proposition 5.10. Let $k$ be an algebraically closed field of characteristic zero and $a, b \in k-\left\{-1,-2^{-1}, 0\right\}$. Then $\mathcal{H}_{a}$ and $\mathcal{H}_{b}$ are isomorphic as Poisson algebras if and only if $a=b$ or $a=-b(2 b+1)^{-1}$. In particular, the factorization index $\left[\mathcal{H}_{1,1,1,1}^{1}: k_{0}\right]^{f}$ is infinite.

Proof. Suppose $\varphi: \mathcal{H}_{a} \rightarrow \mathcal{H}_{b}$ is a Poisson algebra isomorphism, where $\varphi\left(H_{1}\right)=$ $\Sigma_{i=1}^{3} \alpha_{i} H_{i}, \varphi\left(H_{2}\right)=\Sigma_{i=1}^{3} \beta_{i} H_{i}, \varphi\left(H_{3}\right)=\Sigma_{i=1}^{3} \gamma_{i} H_{i}, \alpha_{i}, \beta_{i}, \gamma_{i} \in k$. Thus Theorem 5.9 implies $r_{(0, a)} \sim r_{(0, b)}$ and we obtain:

$$
\begin{gathered}
\gamma_{1}=\gamma_{2}=0, \quad \alpha_{1}^{2}+2 \alpha_{1} \alpha_{2} b=\gamma_{3}, \quad \beta_{1}^{2}+2 \beta_{1} \beta_{2} b=0 \\
\alpha_{1} \beta_{1}+\left(\alpha_{1} \beta_{2}+\alpha_{2} \beta_{1}\right) b=a \gamma_{3}, \quad\left(\alpha_{1} \beta_{2}-\alpha_{2} \beta_{1}\right)(b+1)=(a+1) \gamma_{3}
\end{gathered}
$$

To start with, we point out that since $\varphi$ is an isomorphism we must have $\gamma_{3} \neq 0$. The last part of (5.21) implies $\beta_{1}=0$ or $\beta_{1}+2 \beta_{2} b=0$. Assume first that $\beta_{1}=0$. As $\varphi$ is an isomorphism it follows that $\alpha_{1} \neq 0$. Then the first part of (5.22) comes down to $\beta_{2}=a b^{-1} \alpha_{1}^{-1} \gamma_{3}$. Using the second part of (5.21) we obtain

$$
\beta_{2}=a b^{-1} \alpha_{1}^{-1} \underline{\gamma_{3}} \stackrel{(5.21)}{=} a b^{-1} \alpha_{1}^{-1}\left(\alpha_{1}^{2}+2 \alpha_{1} \alpha_{2} b\right)=a b^{-1}\left(\alpha_{1}+2 \alpha_{2} b\right)
$$

Using the second part of (5.21) and the above formulae for $\beta_{2}$, the last part of (5.22) becomes:

$$
\begin{gathered}
(a+1) \gamma_{3}=\alpha_{1} a b^{-1}\left(\alpha_{1}+2 \alpha_{2} b\right)(b+1)=a b^{-1} \underline{\left(\alpha_{1}^{2}+2 \alpha_{1} \alpha_{2} b\right)}(b+1) \\
\stackrel{(5.21)}{=} a b^{-1} \gamma_{3}(b+1)
\end{gathered}
$$

As $\gamma_{3} \neq 0$ we obtain $a b^{-1}(b+1)=a+1$ which implies $a=b$. Assume now that $\beta_{1} \neq 0$. Therefore, by the last part of (5.21) we get $\beta_{1}=-2 \beta_{2} b$. Now using this formulae for $\beta_{1}$, (5.22) becomes:

$$
-\beta_{2} b\left(\alpha_{1}+2 \alpha_{2} b\right)=a \gamma_{3}, \quad \beta_{2}\left(\alpha_{1}+2 \alpha_{2} b\right)=(a+1)(b+1)^{-1} \gamma_{3}
$$

As $\gamma_{3} \neq 0$, we obtain $-a=b(a+1)(b+1)^{-1}$ which gives $a=-b(2 b+1)^{-1}$. Therefore we proved that $\mathcal{H}_{a}$ and $\mathcal{H}_{b}$ are isomorphic Poisson algebras if and only if $a=b$ or $a=-b(2 b+1)^{-1}$. Together with the fact algebraically closed fields are infinite we obtain $\left[\mathcal{H}_{1,1,1,1}^{1}: k_{0}\right]^{f}$ is infinite. This finishes the proof. 


\section{References}

[1] L. Abrams, The quantum Euler class and the quantum cohomology of the Grassmannians, Is. J. Math., 117(2000), 335-352.Zbl 0954.53048 MR 1760598

[2] A. L. Agore, Classifying complements for associative algebras, Linear Algebra Appl., 446 (2014), 345-355. Zbl 1297.16006 MR 3163149

[3] A. L. Agore and G. Militaru, Extending structures II: the quantum version, $J$. Algebra, 336 (2011), 321-341. Zbl 1264.16031 MR 2802546

[4] A. L. Agore and G. Militaru, Classifying complements for Hopf algebras and Lie algebras, J. Algebra, 391 (2013), 193-208. Zbl 1293.16026 MR 3081628

[5] A. L. Agore and G. Militaru, Extending structures for Lie algebras, Monatsh. für Mathematik, 174 (2014), 169-193. Zbl 06312503 MR 3201255

[6] A. L. Agore and G. Militaru, Bicrossed products, matched pair deformations and the factorization index for Lie algebras, Symmetry Integr. Geom., 10 (2014), 065, 16pp. Zbl 06334468 MR 3226985

[7] A. L. Agore and G. Militaru, Extending structures, Galois groups and supersolvable associative algebras, to appear in Monatsh. für Mathematik. DOI 10.1007/s00605-015-0814-8

[8] C. Bai, Double constructions of Frobenius algebras, Connes cocycles and their duality, J. Noncommu. Geom., 4 (2010), 475-530. Zbl 1250.17028 MR 2718800

[9] N. Bourbaki, Lie groups and Lie algebras, Ch. 1-3, Springer, Paris, 1989. Zbl 0672.22001 MR 0979493

[10] G. Böhm, F. Nill and K. Szlachányi, Weak Hopf algebras. I. Integral theory and C*-structure, J. Algebra, 221 (1999), 385-438. Zbl 0949.16037 MR 1726707

[11] T. Brzeziński and R. Wisbauer, Corings and comodules, London Math. Soc. Lect. Note Ser., 309, Cambridge University Press, 2003. Zbl 1035.16030 MR 2012570

[12] D. Burde and M. Ceballos, Abelian ideals of maximal dimension for solvable Lie algebras, J. Lie Theory, 22 (2012), 741-756. Zbl 1257.17015 MR 3012152

[13] S. Caenepeel, G. Militaru and S. Zhu, Frobenius and Separable Functors for Generalized Module Categories and Nonlinear Equations, Lect. Notes Math., 1787, Springer Verlag, Berlin, 2002. Zbl 1008.16036 MR 1926102

[14] M. Ceballos and D. A. Towers, On abelian subalgebras and ideals of maximal dimension in supersolvable Lie algebras, J. Pure Appl. Algebra, 218 (2014), 497-503. Zbl 1281.17006 MR 3124213

[15] L. Chekhov and M. Mazzocco, Poisson Algebras of Block-Upper-Triangular Bilinear Forms and Braid Group Action, Comm. Math. Physics, 322 (2013), 49-71. Zbl 1296.17008 MR 3073157 
[16] V. Drinfeld, Quantum groups, Proc. Intern. Congr. Math., Berkeley, I, 798-820, 1987. Zbl 0667.16003 MR 0934283

[17] J. M. Figueroa-O'Farrill and S. Stanciu, On the structure of symmetric selfdual Lie algebras, J. Math. Phys., 37 (1996), 4121-4134. Zbl 0863.17004 MR 1400838

[18] F. Gavarini, The crystal duality principle: from Hopf algebras to geometrical symmetries, J. Algebra, 285 (2005), 399-437. Zbl 1064.17011 MR 2119120

[19] V. V. Gorbatsevich, On the level of some solvable Lie algebras, Siberian Math. J., 39 (1998), 872-883. Zbl 0919.17007 MR 1650732

[20] J. Grabowski, Abstract Jacobi and Poisson structures, J. Geom. Phys., 9 (1992), 45-73. Zbl 0761.16012 MR 1154239

[21] J. Grabowski, G. Marmo and A. M. Perelomov, Poisson structures:towards a classification, Modern Phys. Lett. A, 8 (1993), 1719-1733. Zbl 1020.37529 MR 1229646

[22] J. Grabowski and G. Marmo, The graded Jacobi algebras and (co)homology, J. Phys. A, 36 (2003),161-181. Zbl 1039.53090 MR 1959019

[23] J. Grabowski, Brackets, Int. J. Geom. Methods Mod. Phys., 10 (2013), 1360001, 45pp. MR 3092551

[24] J. E. Humphreys, Introduction to Lie algebras and Representation theory, Springer, 1972. Zbl 0254.17004 MR 0323842

[25] M. C. Iovanov, Generalized Frobenius algebras and Hopf algebras, Canad. J. Math., 66 (2014), 205-240. Zbl 1296.16037 MR 3150709

[26] N. Jacobson, Lie Algebras, Interscience Publ., New York-London, 1962. Zbl 0121.27504 MR 0143793

[27] L. Kadison, New Examples of Frobenius Extensions, Univ. Lect. Series, 14, Amer. Math. Soc., Providence, 1999. Zbl 0929.16036 MR 1690111

[28] I. Kath and M. Olbrich, Metric Lie algebras with maximal isotropic centre, Math. Z., 246 (2004), 23-53. Zbl 1046.17003 MR 2031443

[29] J. Kock, Frobenius algebras and $2 D$ topological quantum field theories, London Math. Soc., 59, Cambridge University Press, 2003. Zbl 1046.57001 MR 2037238

[30] A. Kirillov, Local Lie Algebras, Russian Math. Surveys, 31 (1976), 55-75. Zbl 0357.58003 MR 0438390

[31] T. Lambre, G. Zhou and A. Zimmermann, The Hochschild cohomology ring of a Frobenius algebra with semisimple Nakayama automorphism is a BatalinVilkovisky algebra, J. Algebra, 446 (2016), 103-131. MR 3421088 
[32] C. Laurent-Gengoux, A. Pichereau and P. Vanhaecke, Poisson Structures, 347, Springer, 2013. Zbl 1284.53001 MR 2906391

[33] A. Lichnerowicz, Le variétés de Jacobi et leurs algèbres de Lie associées, $J$. Math. Pures Appl., 57 (1978), 453-488. Zbl 0407.53025 MR 0524629

[34] S. Lie, Theorie der Transformationsgruppen, 1-3, Leipzig, 1888-93. Zbl 20.0368.01 (1888); Zbl 23.0364.01 (1890); Zbl 25.0623.01 (1893)

[35] J. Lu, X. Wang and G. Zhuang, Universal enveloping algebras of Poisson Hopf algebras, J. Algebra, 426 (2015), 92-136. Zbl 06399159 MR 3301903

[36] J. H. Lu and A. Weinstein, Poisson Lie groups, dressing transformations and Bruhat decompositions, J. Differential Geom., 31 (1990), 501-526. Zbl 0673.58018 MR 1037412

[37] S. Majid, Physics for algebraists: non-commutative and non-cocommutative Hopf algebras by a bicrossproduct construction, J. Algebra, 130 (1990), 17-64. Zbl 0694.16008 MR 1045735

[38] C. M. Marle, On Jacobi Manifolds and Jacobi boundles, in Symplectic Geometry, Groupoids, and Integrable Systems, 227-246, Mathematical Sciences Research Institute Publications, 20, Springer, 1991. Zbl 0735.53023 MR 1104931

[39] G. Mazzola, The algebraic and geometric classification of associative algebras of dimension five, Manuscripta Math., 27 (1979), 81-101. Zbl 0446.16033 MR 0524979

[40] A. Medina and Ph. Revoy, Algébres de Lie et produit scalaire invariant, Ann. Sci. Ècole Norm. Sup., 18 (1985), 553-561. Zbl 0592.17006 MR 0826103

[41] Xiang Ni and Chengming Bai, Poisson bialgebras, J. Math. Phys., 54 (2013), 023515. Zbl 1290.17019 MR 3076642

[42] O. Pelc, A New Family of Solvable Self-Dual Lie Algebras, J. Math. Phys., 38 (1997), 3832-3840. Zbl 0897.17009 MR 1455588

[43] R. O. Popovych, V. M. Boyko, M. O. Nesterenko and M. W. Lutfullin, Realizations of real low-dimensional Lie algebras, Journal of Physics A: Mathematical and General, 36 (2003), 7337. Zbl 1040.17021 MR 2004893

[44] D. E. Radford, Hopf algebras, Series on knots and everything, 49, World Scientific Publishing, 2012. Zbl 1266.16036 MR 2894855

[45] A. Rovi, Hopf algebroids associated to Jacobi algebras, Int. J. Geom. Methods Mod. Phys., 11 (2014), 1450092, 20pp. MR 3275818

[46] E. Study, Über Systeme complexer Zahlen und ihre Anwendungen in der Theorie der Transformationsgruppen, Monatshefte für Mathematik, 1 (1890), 283-354. Zbl 22.0387.02 MR 1546158 
[47] C. Zhu, F. van Oystaeyen and Y. Zhang, On the (co)homology of Frobenius Poisson algebras, Journal of K-theory: K-theory and its Applications to Algebra, Geometry, and Topology, 14 (2014), 371-386. Zbl 1309.17012 MR 3264266

Received 11 September, 2014

A. L. Agore, Faculty of Engineering, Vrije Universiteit Brussel, Pleinlaan 2,

B-1050 Brussels, Belgium;

"Simion Stoilow" Institute of Mathematics of the Romanian Academy, P.O. Box 1-764, 014700 Bucharest, Romania

E-mail: ana.agore@vub.ac.be; ana.agore@gmail.com

G. Militaru, Faculty of Mathematics and Computer Science, University of Bucharest, Str. Academiei 14, RO-010014 Bucharest 1, Romania

E-mail: gigel.militaru@fmi.unibuc.ro; gigel.militaru@gmail.com 